\title{
EXTENDED CANONICAL ALGEBRAS AND FUCHSIAN SINGULARITIES
}

\author{
HELMUT LENZING AND JOSÉ A. DE LA PEÑA
}

\section{Introduction}

1.1. Main results. Let $k$ be any field. Consider the hereditary algebra $A=k \Delta$ associated to a finite connected quiver without oriented cycles. A fundamental fact in Representation Theory of Algebras is the distinction of the representation type of $A$ : $A$ is representation-finite (that is, there are only finitely many indecomposable $A$-modules, up to isomorphism) exactly when the underlying graph $|\Delta|$ of $\Delta$ is of Dynkin type; $A$ is tame (that is, for each $d \in \mathbb{N}$, the indecomposable $d$-dimensional $A$-modules may be classified in a finite number of one-parameter families of modules) exactly when $|\Delta|$ is of extended Dynkin type; in the remaining cases $A$ is wild (that is, there is a embedding $\bmod -k\langle x, y\rangle \rightarrow \bmod -A$ which preserves indecomposability and isomorphism classes from the category of finite dimensional modules over the ring in two non-commuting indeterminates into the category of finite dimensional $A$ modules). Consider the Auslander-Reiten translation $\tau_{A}$ in mod- $A$ and $P$ an indecomposable projective $A$-module. In case $A$ is tame representation-infinite, the sequence of modules $\left(\tau_{A}^{-n} P\right)_{n}$ is well defined and the algebra $R(A, P)=\bigoplus_{n=0}^{\infty} \operatorname{Hom}_{A}\left(P, \tau_{A}^{-n} P\right)$ is an infinite dimensional positively $\mathbb{Z}$-graded surface singularity. The algebra $R(A, P)$ reflects many properties of $A$ and $P$ and has a particularly interesting structure, as shown in [12]:

Assume $A=\mathbb{C} \tilde{\Delta}$ is a tame hereditary algebra where $\tilde{\Delta}$ extends the Dynkin type $|\Delta|$. Let $P$ the indecomposable projective associated to the vertex in $\tilde{\Delta} \backslash \Delta$. Then $R(A, P)$ is isomorphic to the algebra of invariants $\mathbb{C}[x, y]^{G}$, where $G \subset S L(2, \mathbb{C})$ is a binary polyhedral group of type $|\Delta|$. Accordingly the completion of the graded algebra $R(A, P)$ is isomorphic to the surface singularity of type $|\Delta|$.

In [34], Ringel introduced the canonical algebras $C=C(p, \lambda)$ depending on a weight sequence $p=\left(p_{1}, \ldots, p_{t}\right)$ of positive integers and a parameter sequence $\lambda=$ 
$\left(\lambda_{3}, \ldots, \lambda_{t}\right)$ of pairwise distinct non-zero elements from $k$. In [11] it was shown that $\bmod -C$ is derived equivalent to $\operatorname{coh}(\mathbb{X})$ the category of coherent sheaves on a weighted projective line $\mathbb{X}=\mathbb{X}(p, \lambda)$.

One of the aims of this work is the introduction of a class of algebras with related interesting properties. Let $P$ be an indecomposable projective module over a canonical algebra $C=C(p, \lambda)$, the one-point extension $A=C[P]$ defined as the matrix algebra

$$
\left[\begin{array}{ll}
k & 0 \\
P & C
\end{array}\right]
$$

is called an extended canonical algebra. In section 2 we show that for two indecomposable projective $C$-modules $P$ and $P^{\prime}$, the algebras $C[P]$ and $C\left[P^{\prime}\right]$ are derived equivalent. Moreover, if $C$ is of tame type, the extended canonical algebra is derived equivalent to a wild hereditary or a wild canonical algebra, so essentially $C[P]$ belongs to a well-studied class of algebras. There are interesting phenomena arising when $C$ (and hence $A$ ) is of wild type.

Consider the Coxeter transformation of $A$ as an automorphism $\varphi_{A}: K_{0}(A) \rightarrow$ $K_{0}(A)$ of the Grothendieck group of $A$, given on the classes of indecomposable projective modules by the formula $\varphi_{A}([P(S)])=-[I(S)]$, where $P(S)$ (resp. $I(S)$ ) is the projective cover (resp. injective envelope) of a simple module $S$. The characteristic polynomial $f_{A}(T)$ of $\varphi_{A}$ is called the Coxeter polynomial of $A$.

Let $\chi_{\mathbb{X}}=2-\sum_{i=1}^{t}\left(1-1 / p_{i}\right)$ be the (orbifold) Euler characteristic of $\mathbb{X}$. As shown in [12], for $\chi_{\mathbb{X}}>0$ the classification problem of $\operatorname{coh}(\mathbb{X})$ is related to the problem of classifying the Cohen-Macaulay modules over a simple surface singularity and is in fact equivalent to the problem of classifying the graded Cohen-Macaulay modules over a corresponding quasi-homogeneous singularity. Assume $\chi_{\mathbb{X}}<0$. By [23, 25] we know that for $A=C[P]$ an extended canonical algebra

$$
f_{A}(T)=P_{C}(T) f_{C}(T)
$$

where $P_{C}(T)$ is the Hilbert-Poincare series of the positively graded algebra $R(p, \lambda)=$ $\bigoplus_{n=0}^{\infty} \operatorname{Hom}_{C}\left(M, \tau_{C}^{n} M\right)$, where $M$ is a rank one not preprojective $C$-module. Equivalently, $R(p, \lambda)=\bigoplus_{n=0}^{\infty} \operatorname{Hom}\left(\mathcal{O}, \tau_{\mathbb{X}}^{n} \mathcal{O}\right)$, where $\mathcal{O}$ is the structure sheaf on $\mathbb{X}$. Recall from [21, 23] that in case $k=\mathbb{C}$, we can interpret $R(p, \lambda)$ as an algebra of entire automorphic forms associated to the action of a suitable Fuchsian group of the first kind, acting on the upper half plane $\mathbb{H}_{+}$. 
¿From [21], we know that the $k$-algebra $R=R(p, \lambda)$ is commutative, graded integral Gorenstein, in particular Cohen-Macaulay, of Krull dimension two. The complexity of the surface singularity $R$ is described by the triangulated category

$$
\mathrm{D}_{\mathrm{Sg}}^{\mathbb{Z}}(R)=\frac{\mathrm{D}^{b}\left(\bmod ^{\mathbb{Z}}-R\right)}{\mathrm{D}^{b}\left(\operatorname{proj}^{\mathbb{Z}}-R\right)},
$$

where $\bmod ^{\mathbb{Z}}-R$ (resp. $\operatorname{proj}^{\mathbb{Z}}-R$ ) denotes the category of finitely generated (resp. finitely generated projective) $\mathbb{Z}$-graded $R$-modules. This category was considered by Buchweitz [5] and Orlov [30], see also Krause's account [19] for a related, but slightly different approach. For $\chi_{\mathbb{X}}>0$, where the weight type of $\mathbb{X}$ determines a Dynkin quiver $\Delta$, Kajiura, Saito, Takahashi and Ueda [17] have shown that $\mathrm{D}_{\mathrm{Sg}}^{\mathbb{Z}}(R)$ is equivalent to the derived category of finite dimensional modules over the path algebra $k \Delta$. For $\chi_{\mathbb{X}}=0$, the algebra $R$ hence $\mathrm{D}_{\mathrm{Sg}}^{\mathbb{Z}}(R)$ is not defined, but a close variant $\mathrm{D}_{\mathrm{Sg}}^{\mathbb{L}(p)}(S)$, as shown by Ueda [35], is equivalent to the derived category $\mathrm{D}^{b}(\operatorname{coh}(\mathbb{X}))$ of coherent sheaves on a weighted projective line which is tubular, that is, has weight type $(2,3,6),(2,4,4),(3,3,3)$ or $(2,2,2,2)$.

In section 3 we deal with the case $\chi_{\mathbb{X}}<0$ and prove that this category, as first observed by Saito and Takahashi (for the field $\mathbb{C}$ of complex numbers), is described in the following way.

Theorem 1. Let $k$ be an algebraically closed field. Assume $\chi_{\mathbb{X}}<0$ and let $R$ be the positively $\mathbb{Z}$-graded surface singularity attached to $\mathbb{X}$. Then there exists a tilting object $\bar{T}$ in the triangulated category $\mathrm{D}_{\mathrm{Sg}}^{\mathbb{Z}}(R)$ whose endomorphism ring is isomorphic to an extended canonical algebra $C[P]$, where $C$ is the canonical algebra associated with $\mathbb{X}$.

It follows that the categories $\mathrm{D}_{\mathrm{Sg}}^{\mathbb{Z}}(R)=\mathcal{T}$ and $\mathrm{D}^{b}(\bmod (C[P]))$ are equivalent as triangulated categories. In section 3.9 we further introduce the concept of CoxeterDynkin algebras and establish their relationship to the Coxeter-Dynkin diagrams from singularity theory.

More precise information on the structure of the ring $R(p, \lambda)$ is obtained by a closer examination of the spectral properties of the Coxeter transformation of the extended canonical algebra $C[P]$.

A sequence of weights $p=\left(p_{1}, \ldots, p_{t}\right)$ will always satisfy $p_{1} \leq p_{2} \leq \cdots \leq p_{t}$. We consider the lexicographical ordering of sequences $\left(p_{1}, \ldots, p_{t}\right) \leq\left(q_{1}, \ldots, q_{s}\right)$ if $t=s$ 
and $p_{i} \leq q_{i}$ for $1 \leq i \leq t$. Extend the relation $p \leq q$ (and say that $q$ dominates $p)$ to weight sequences of (possibly) different length by adding 1's if necessary. The following result is shown in section 4 based on techniques developed in [24] and will be fundamental in the proof of the main results.

Theorem 2. Let $A=C[P]$ be an extended canonical algebra of the wild canonical algebra $C=C(p, \lambda)$. The following happens:

(a) $f_{A}(T)$ has at most 4 roots not in $\mathbb{S}^{1}$.

(b) The roots of $f_{A}(T)$ lie on the unit circle $\mathbb{S}^{1}$ if and only if the weight sequence $p$ belongs to the 38-member list determined by all $p<q$ with $q$ belonging to the following critical list:

$$
\begin{aligned}
(t=3): & (2,3,11),(2,4,9),(2,5,8),(2,6,7), \\
& (3,3,8),(3,4,7),(3,5,6), \\
& (4,4,6),(4,5,5), \\
(t=4): & (2,2,2,7),(2,2,3,6),(2,3,4,4),(3,3,3,4), \\
(t=5): & (2,2,2,2,5),(2,2,2,3,4),(2,2,3,3,3), \\
(t=6): & (2,2,2,2,2,3), \\
(t=7): & (2,2,2,2,2,2,2) .
\end{aligned}
$$

We shall say that the algebra $R(p, \lambda)$ (and also the weight sequence $p$ ) is formally $n$-generated if

$$
P_{C}(T)=\frac{\prod_{i=1}^{n-2}\left(1-T^{c_{i}}\right)}{\prod_{j=1}^{n}\left(1-T^{d_{j}}\right)}
$$

for certain natural numbers $c_{1}, \ldots, c_{n-2}$ and $d_{1}, \ldots, d_{n}$, all $\geq 2$. The algebra $R(p, \lambda)$ (and also the weight sequence $p$ ) is formally a complete intersection if $P_{C}(T)$ is a rational function $f_{1}(T) / f_{2}(T)$, where each $f_{i}(T)$ is a product of cyclotomic polynomials.

Theorem 3. Let $C=C(p, \lambda)$ be a wild canonical algebra with weight sequence $\left(p_{1}, \ldots, p_{t}\right)$ and $A=C[P]$ be an extended canonical algebra. The following are equivalent:

(a) $R(p, \lambda)$ is formally 3 - or 4-generated

(b) $R(p, \lambda)$ is formally a complete intersection 
(c) The roots of $f_{A}(T)$ lie on $\mathbb{S}^{1}$.

Moreover, for $t=3$ the algebra $R(p, \lambda)$ is a graded complete intersection of the form $k\left[X_{1}, \ldots, X_{s}\right] /\left(\rho_{3}, \ldots, \rho_{s}\right)$ where $s=3$ or 4 and $\rho_{3}, \ldots, \rho_{s}$ is a homogeneous regular sequence. For $k=\mathbb{C}$ the assertion also holds for $t \geq 4$ for $R\left(p, \lambda^{\prime}\right)$ for a suitable choice of parameters. $\lambda^{\prime}=\left(\lambda_{3}^{\prime}, \ldots, \lambda_{t}^{\prime}\right)$.

We remark that in almost every case Root $f_{A}(T) \subset \mathbb{S}^{1}$ implies that the Coxeter transformation $\varphi_{A}$ is periodic. In fact the weight sequences $(3,3,3,3)$ and $(2,2,2,2,4)$ are the only exceptions (section 4).

Theorem 4. Let $C=C(p, \lambda)$ be a wild canonical algebra with weight sequence $p=$ $\left(p_{1}, \ldots, p_{t}\right)$. Consider $A=C[P]$ an extended canonical algebra. The following are equivalent:

(a) $R(p, \lambda)$ is formally 3-generated

(b) $\varphi_{A}$ is periodic of period $d$ and there is a primitive $d$-th root of unity which is root of $f_{A}(T)$.

For $t=3$ the algebra $R(p, \lambda)$ is always a graded complete intersection of the form $k\left[X_{1}, X_{2}, X_{3}\right] /(f)$. Moreover, for $t \geq 4$ and $k=\mathbb{C}$ this also holds for $R\left(p, \lambda^{\prime}\right)$ for a suitable choice of parameters $\lambda^{\prime}=\left(\lambda_{3}^{\prime}, \ldots, \lambda_{t}^{\prime}\right)$.

For the proof of Theorem 3 (resp. Theorem 4) we classify in section 5 all the weight sequences $p$ such that $R(p, \lambda)$ is formally a complete intersection (resp. $R(p, \lambda)$ has 3 homogeneous generators). In the complex case the algebras $R(p, \lambda)$ of Theorem 3 correspond to the Fuchsian singularities which are minimal elliptic [36, Proposition 5.5.1] and the classification is related to Laufer's [20]. The algebras $R(p, \lambda)$ of Theorem 4 relate to classifications by Dolgachev [6] and Wagreich [37] and include the 14 exceptional unimodal Arnold's singularities [1]. We refer the reader to the complete account by Ebeling [9].

The research for this work was done during exchange visits México-Paderborn. We thank our universities and CONACyT in México for support. We thank Henning Krause for directing our attention to Buchweitz's paper [5]. 
1.2. Notation and conventions. Let $Q$ be a finite quiver without oriented cycles. The path algebra $k Q$ has as basis all the oriented paths in $Q$ and product given by juxtaposition of paths. Given an ideal $I$ of $k Q$ which is admissible (that is, $\left(k Q^{+}\right)^{m} \subset I \subset\left(k Q^{+}\right)^{2}$ for some $m \geq 2$, where $k Q^{+}$is the ideal of $k Q$ generated by the arrows), we consider the finite dimensional $k$-algebra $A=k Q / I$. By 'module' we mean a finite dimensional right $A$-module. The category of modules is denoted $\bmod -A$. A module is identified with a covariant functor $X: k Q \rightarrow \bmod -k$ such that $X(\rho)=0$ for every $\rho \in I$. Important modules are the simple modules $S_{i}$ associated to vertices $i \in Q_{0}\left(=\right.$ set of vertices of $Q$ ), the projective cover $P_{i}=A(-, i)$ of $S_{i}$ and the injective envelope $I_{i}=D A(i,-)$ of $S_{i}$, where $D=\operatorname{Hom}_{k}(-, k)$ is the canonical duality.

We denote by $K_{0}(A)$ the Grothendieck group of $A$. Since $A$ has finite global dimension, the classes $\left[P_{i}\right]$ with $i \in Q_{0}$ form a basis of $K_{0}(A)$. Thus the Coxeter transformation $\varphi_{A}: K_{0}(A) \rightarrow K_{0}(A)$, given by $\varphi_{A}\left(\left[P_{i}\right]\right)=-\left[I_{i}\right]$ defines an isomorphism. The Grothendieck group $K_{0}(A)$ is equipped with a bilinear form $\langle-,-\rangle_{A}: K_{0}(A) \times K_{0}(A) \rightarrow \mathbb{Z}$, called the Euler form, defined in the classes of modules $X$ and $Y$ as $\langle[X],[Y]\rangle_{A}=\sum_{i=0}^{\infty}(-1)^{i} \operatorname{dim}_{k} \operatorname{Ext}_{A}^{i}(X, Y)$. In case $A=k \Delta$ is a hereditary algebra, then $\varphi_{A}([X])=\left[\tau_{A} X\right]$ for any indecomposable non-projective $A$-module $X$. For the general situation, we have to look at the derived category $D(A)=\mathrm{D}^{b}(\bmod -A)$ of bounded complexes of $A$-modules.

The derived category $D(A)$ contains a copy $\bmod -A[n]$ of $\bmod -A$ for each integer $n \in \mathbb{Z}$, with objects written $X[n]$ and satisfying

$$
\operatorname{Hom}_{D(A)}(X[n], Y[m])=\operatorname{Ext}_{A}^{n-m}(X, Y) .
$$

We say that an algebra $A$ is derived hereditary (resp. derived canonical) if $D(A)$ is triangle equivalent to $D(H)$ (resp. $D(C)$ ) for a hereditary algebra $H$ (resp. a canonical algebra $C$ ).

The category $D(A)$ has Auslander-Reiten triangles which yield a self-equivalence $\tau_{D(A)}$ of $D(A)$, the Auslander-Reiten translation, satisfying $\operatorname{Hom}_{D(A)}\left(Y, \tau_{D(A)} X[1]\right)=$ $D \operatorname{Hom}_{A}(X, Y)$. The natural isomorphism $K_{0}(A) \rightarrow K_{0}(D(A)), X \mapsto X[0]$ yields $\varphi_{A}([X])=\left[\tau_{D(A)} X\right]$.

For background material on representations of algebras and derived categories we refer the reader to [14, 34].

For vectors $v, w \in K_{0}(A)$ we get $\left\langle v, \varphi_{A}(w)\right\rangle_{A}=-\langle w, v\rangle_{A}$. 


\section{Extended canonical algebras: basic properties}

2.1. Let $C=C(p, \lambda)$ be the canonical algebra defined by the weight sequence $p=$ $\left(p_{1}, \ldots, p_{t}\right)$ with $p_{i} \geq 2$ and $\left(\lambda=\lambda_{3}, \ldots, \lambda_{t}\right)$ a sequence of pairwise distinct non-zero elements of $k$, that is, $C$ is defined by the quiver

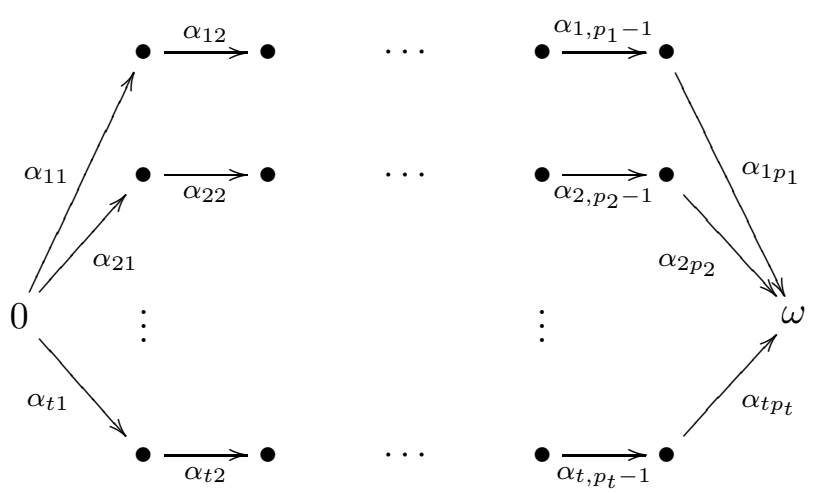

satisfying the $t-2$ equations

$$
\alpha_{i p_{i}} \ldots \alpha_{i 2} \alpha_{i 1}=\alpha_{2 p_{2}} \ldots \alpha_{22} \alpha_{21}-\lambda_{i} \alpha_{1 p_{1}} \ldots \alpha_{12} \alpha_{11}, i=3, \ldots, t .
$$

The algebra $C$ is a one-point extension $H[M]$ of the hereditary algebra $H=C /(0)$ by an $H$-module $M$ with dimension vector $[M]=\left(\operatorname{dim}_{k} M(i)\right)_{i} \in K_{0}(H)$ as follows:

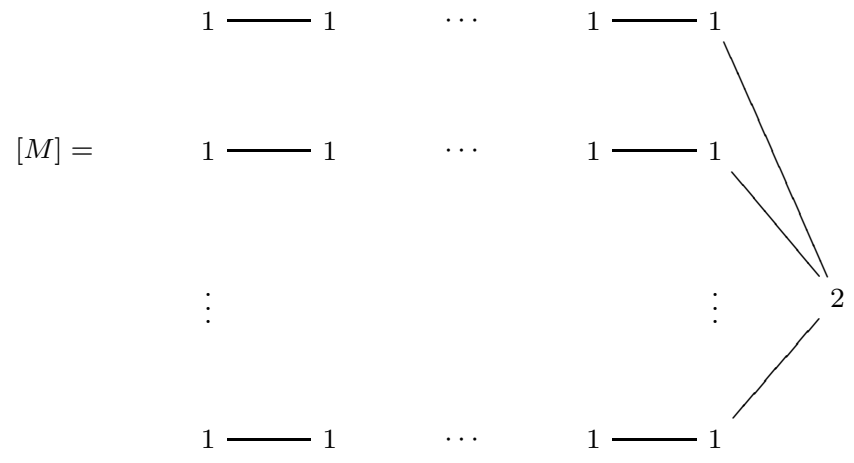

In case $t \geq 3$, the module $M$ is an indecomposable $H$-module which is not preprojective or preinjective.

Observe that the underlying graph of $H$ is a star $\left[p_{1}, p_{2}, \ldots, p_{t}\right]$ with linear arms having $p_{i}$ vertices, $i=1, \ldots, t$. If $H$ is representation-finite, then $\left[p_{1}, p_{2}, \ldots, p_{t}\right]$ is a Dynkin diagram (that is, $\sum_{i=1}^{t} \frac{1}{p_{i}}>t-2$ ) and $C$ is tame of domestic type. If $H$ is tame, then $\left[p_{1}, p_{2}, \ldots, p_{t}\right]$ is an extended Dynkin diagram (that is, $\sum_{i=1}^{t} \frac{1}{p_{i}}=t-2$ ) and $C$ is tame of tubular type. See [34] for details. 
2.2. The representation theory of $\bmod -C$ for $C=C(p, \lambda)$ a canonical algebra is controlled by the category $\operatorname{coh}(\mathbb{X})$ of coherent sheaves on a weighted projective line $\mathbb{X}=\mathbb{X}(p, \lambda)$, since the derived categories $D(C)$ and $\mathrm{D}^{b}(\operatorname{coh}(\mathbb{X}))$ are equivalent as triangulated categories [12]. The complexity of the classification problem for coh $(\mathbb{X})$, and hence for mod- $C$, is essentially determined by the (orbifold) Euler characteristic $\chi_{\mathbb{X}}=2-\sum_{i=0}^{t}\left(1-1 / p_{i}\right)$. Indeed, for $\chi_{\mathbb{X}}>0$, the algebra $C$ is tame of domestic type and for $\chi_{\mathbb{X}}=0$, the algebra $C$ is tubular. The wild case $\chi_{\mathbb{X}}<0$ was carefully studied in [23], a paper which is the basis of the present investigation.

2.3. Let $C=C(p, \lambda)$ be a canonical algebra. Let $P$ be an indecomposable projective or injective $C$-module, then $A=C[P]$ is called an extended canonical algebra. Hence $A$ arises from by adjoining one arrow with a new vertex to an arbitrary vertex of $C$ while keeping the relations for $C$ without introducing any new relations. In particular, the opposite algebra of an extended canonical algebra is again extended canonical.

Lemma. Any extended canonical algebra is wild.

Proof: Recall that for an algebra $B=k Q / I$ where $Q$ has no oriented cycles and $I$ is generated by $\rho_{1}, \ldots, \rho_{s} \in \bigcup_{i, j \in Q_{0}} I(i, j)$, the Tits (quadratic) form $q_{B}: K_{0}(B) \rightarrow \mathbb{Z}$ is defined by

$$
q_{B}(x)=\sum_{i \in Q_{0}} x(i)^{2}-\sum_{i \rightarrow j} x(i) x(j)+\sum_{i, j \in Q_{0}} r(i, j) x(i) x(j),
$$

where $r(i, j)=\#\left\{s: \rho_{s} \in I(i, j)\right\}$. The Tits form is weakly non-negative (i.e. $q_{A}(v) \geq$ 0 for $\left.v \in \mathbb{N}^{Q_{0}}\right)$ if $B$ is a tame algebra [31].

For an extended canonical algebra $A=C[P]$ with extension vertex $*$ such that $\operatorname{rad} P_{*}=P=P_{j}$ for some vertex $j$ in $C$, we have the following:

- since $g l \operatorname{dim} A=2$, then $q_{A}(x)=\langle x, x\rangle_{A}$;

- the vector $w \in K_{0}(C) \subset K_{0}(A)$ with $w(i)=1$ for every $i$ in $C$, satisfies $q_{c}(w)=0$

- for $e_{*}=\left[S_{*}\right]$, we get

$$
q_{A}\left(2 w+e_{*}\right)=4 q_{c}(w)-2 w(j)+1<0 .
$$

Hence $A$ is of wild type. 
2.4. The following result is fundamental for introducing the concept of extended canonical algebras.

Proposition. Let $X$ and $Y$ be two indecomposable projective or injective $C$-modules over a canonical algebra $C$. Then the extended canonical algebras $C[X]$ and $C[Y]$ are derived equivalent.

In particular, the derived class of an extended canonical algebra is independent of the chosen projective module.

Proof. (See [24]). Under the equivalence $D(C)=\mathrm{D}^{b}(\operatorname{coh}(\mathbb{X}(p, \lambda)))$ the modules $X$ and $Y$ become line bundles over $\mathbb{X}$ (up to translation in $\mathrm{D}^{b}(\operatorname{coh}(\mathbb{X}))$ ). Since the Picard group of $\mathbb{X}$ acts transitively on isomorphism classes of line bundles, there is a self-equivalence of $D(C)$ sending $X$ to $Y$. The assertion follows from [2].

2.5. The following remark is useful:

Proposition. Let $A$ be an extended canonical algebra of $C=C(p, \lambda)$ with $p=$ $\left(p_{1}, \ldots, p_{t}\right)$ satisfying $t \geq 3$. Then $A$ is derived equivalent to a one-point extension $H[N]$ of a hereditary algebra $H$ by an indecomposable module $N$.

Proof. By (2.3), we may assume that $A$ is the path algebra of the quiver
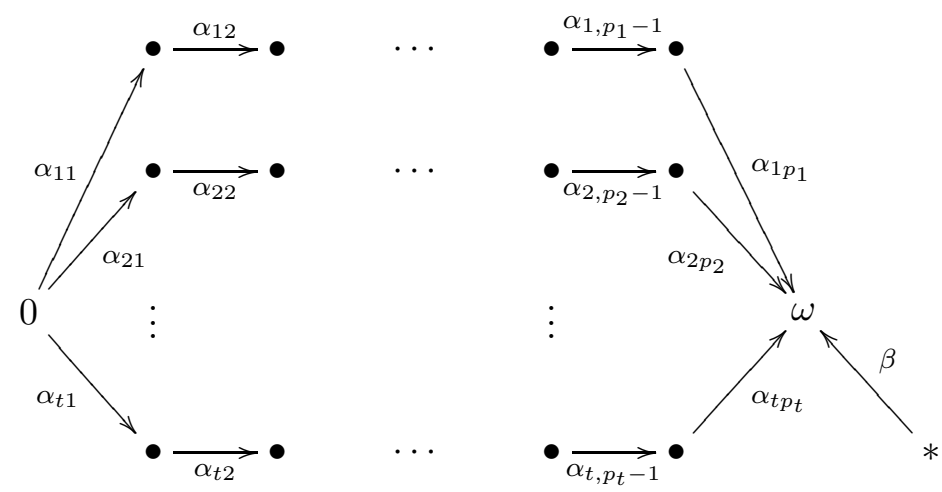

equipped with the canonical relations $\alpha_{i p_{i}} \ldots \alpha_{i 1}=\alpha_{2 p_{2}} \ldots \alpha_{21}-\lambda_{i} \alpha_{1 p_{1}} \ldots \alpha_{11}(3 \leq$ $i \leq t)$. It follows that $A$ is the one-point extension of the path algebra of the star $\left[2, p_{1}, p_{2}, \ldots, p_{t}\right]$ by an indecomposable module $N=N\left(\lambda_{3}, \ldots, \lambda_{t}\right)$ whose restriction to $\left[p_{1}, p_{2}, \ldots, p_{t}\right]$ is $M$ as in $(2.1)$ and $N(*)=0$. 
Remark: An obvious variant of the above statement yields families of one-point extensions of hereditary algebras which are pairwise derived equivalent. For instance, for the canonical type $(2,3,7)$, the Proposition yields $10=(2-1)+(3-1)+(7-1)+1$ choices of pairs $(H, N)$ of a hereditary algebra $H$ and an indecomposable $H$-module $N$ such that $H[N]$ is derived equivalent to an extended canonical algebra of type $(2,3,7)$.

\section{The derived category of an extended canonical algebra}

3.1. In this section we are going to investigate the nature of the bounded derived category of an extended canonical algebra $A=C[P]$. As it turns out the structure of this triangulated category will sensibly depend on the sign of the (orbifold) Euler characteristic $\chi_{\mathbb{X}}$ of the weighted projective line $\mathbb{X}$ associated to $C$.

Let $\mathcal{T}$ be a triangulated $k$-category, see [13, 27, 18] for definition and properties. An object $E$ in $\mathcal{T}$ is called exceptional if $\operatorname{End}(E)=k$ and $\operatorname{Hom}(E, E[n])=0$ for all integers $n \neq 0$. By ${ }^{\perp} E$ (resp. $E^{\perp}$ we denote the full triangulated subcategory of $\mathcal{T}$ consisting of all objects $X \in \mathcal{T}$ (resp. $Y \in \mathcal{T}$ ) satisfying $\operatorname{Hom}(X, E[n])=0$ (resp. $\operatorname{Hom}(E[n], Y)=0$ for each integer $n$. By $\left[3\right.$ the inclusion ${ }^{\perp} E \hookrightarrow \mathcal{T}$ (resp. $E^{\perp} \hookrightarrow \mathcal{T}$ ) admits an exact left (resp. right) adjoint $\ell: \mathcal{T} \rightarrow{ }^{\perp} E$ (resp. $r: \mathcal{T} \rightarrow E^{\perp}$ ).

For the purpose of this paper we call an exceptional object special in $\mathcal{T}$ if one of the following two conditions is satisfied:

(i) the left perpendicular category ${ }^{\perp} E$ is equivalent to $D^{b}(\operatorname{coh}(\mathbb{X}))$ for some weighted projective line $\mathbb{X}$ and, moreover, the left adjoint $\ell$ maps $E$ to a line bundle in $\operatorname{coh}(\mathbb{X})$.

(ii) the right perpendicular category $E^{\perp}$ is equivalent to $\mathrm{D}^{b}(\operatorname{coh}(\mathbb{X}))$ for some weighted projective line $\mathbb{X}$ and, moreover, the right adjoint $r$ maps $E$ to a line bundle in $\operatorname{coh}(\mathbb{X})$.

Again, for the purpose of this paper, an object $T$ of $\mathcal{T}$ is called a tilting object in $\mathcal{T}$ if $(i) T$ generates $\mathcal{T}$ as a triangulated category, $(i i) \operatorname{Hom}(T, T[n])=0$ holds for each non-zero integer $n$, and (iii) the endomorphism algebra of $T$ has finite global dimension.

3.2. Our main tool to investigate the shape of $D(A)$ is the following proposition.

Proposition. Let $\mathcal{T}$ be a triangulated category having an exceptional object $E$ that is special in $\mathcal{T}$. Then there exists a tilting object $\bar{T}$ of $\mathcal{T}$ whose endomorphism ring is an extended canonical algebra. 
Proof. With the previous notations we assume that $E^{\perp}=\mathrm{D}^{b}(\operatorname{coh}(\mathbb{X}))$ and $r(E)$ is a line bundle in $\operatorname{coh}(\mathbb{X})$. (The assumption on the left perpendicular ${ }^{\perp} E$ category is treated similarly.) We choose a tilting object $T$ in $\operatorname{coh}(\mathbb{X})$, hence in $\mathrm{D}^{b}(\operatorname{coh}(\mathbb{X}))$ having the line bundle $r(E)$ as a direct summand and such that $\operatorname{End}(T)=C$ is the canonical algebra attached to $\mathbb{X}$ (see [1] ). We claim that $\bar{T}=T \oplus E$ is a tilting object in $\mathcal{T}$. Indeed, since $T$ generates $\mathrm{D}^{b}(\operatorname{coh} \mathbb{X})$ and $E$ together with $E^{\perp}$ generates $\mathcal{T}$, it follows that $\bar{T}$ generates $\mathcal{T}$. Next, we show that $\operatorname{Hom}(\bar{T}, \bar{T}[n])=0$ for each nonzero integer $n$. This reduces to show that $\operatorname{Hom}(E[n], T)=0$ and $\operatorname{Hom}(T, E[n])$ holds for every nonzero $n$. The first assertion holds for each $n$ since $T$ belongs to $E^{\perp}$. For the second we use that $\operatorname{Hom}(T, E[n])=\operatorname{Hom}(T[-n], r(E))$ is zero since by construction $r(E)$ is a direct summand of the tilting object $T$. Finally, the endomorphism ring of $\bar{T}$ is given as the matrix ring

$$
\left(\begin{array}{cc}
C & 0 \\
P & k
\end{array}\right), \quad \text { where } P=\operatorname{Hom}(T, E)=\operatorname{Hom}(T, r E)
$$

is an indecomposable projective $C$-module, hence $\operatorname{End}(\bar{T})=C[P]$ is an extended canonical algebra. Moreover, as it is easily seen, $C[P]$ has global dimension two.

Keeping the assumptions on $E$ and $\mathcal{T}$ from the proposition, we obtain.

Corollary. If $\mathcal{T}$ is triangle equivalent to a bounded derived category $D(B)$ for some finite dimensional k-algebra $B$ or, more generally, if $\mathcal{T}$ is algebraic in the sense of Keller [18], then $\mathcal{T}$ is triangle equivalent to $\mathrm{D}^{b}(\bmod -A)$, for the extended canonical algebra $A=C[P]$.

Proof. The first claim follows from [32], the general version requires [18] or [4].

3.3. Positive Euler characteristic: the domestic case. Consider a canonical algebra $C=C\left(p_{1}, \ldots, p_{t}\right)$ of domestic type, that is, $\sum_{i=1}^{t} \frac{1}{p_{i}}>1$. Let $\Delta=\left[p_{1}, \ldots, p_{t}\right]$ be the star corresponding to the weight sequence $p=\left(p_{1}, \ldots, p_{t}\right)$ and $\tilde{\Delta}$ be the corresponding extended Dynkin diagram. Then $\tilde{\Delta}$ admits a unique positive additive function $\lambda$ assuming value 1 , that is, $\lambda: \tilde{\Delta} \rightarrow \mathbb{N}$ satisfies the conditions:

(i) $2 \lambda(i)=\sum_{j \in i^{+}} \lambda(j)$, where $i^{+}$is the set of neighbors of $i$;

(ii) for some vertex $i \in \tilde{\Delta}_{0}, \lambda(i)=1$. Such an $i$ is called an extension vertex. 
The double extended graph of type $\Delta$, denoted by $\tilde{\tilde{\Delta}}$, is the graph arising form $\tilde{\Delta}$ by adjoining a new edge in an extension vertex. The list of double extended Dynkin graphs is the following:

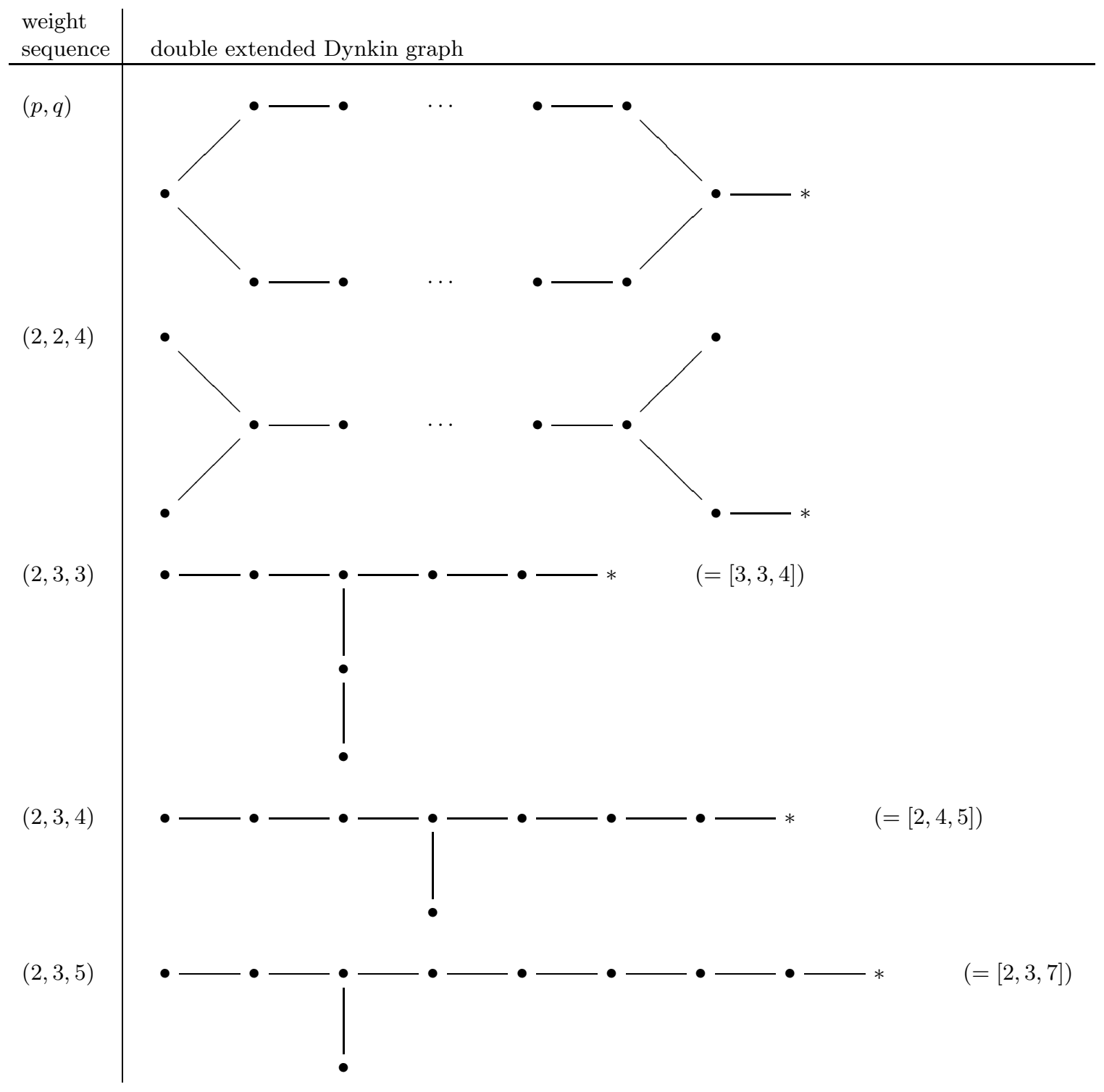

Proposition. Let $C=C(p, \lambda)$ be a canonical algebra of domestic type $p=\left(p_{1}, p_{2}, p_{3}\right)$. Let $\Delta=\left[p_{1}, p_{2}, p_{3}\right]$ be the associated Dynkin diagram. For any indecomposable preprojective $C$-module $N$, the one-point extension $A=C[N]$ is derived equivalent to a hereditary algebra of type $\tilde{\tilde{\Delta}}$. In particular, an extended canonical algebra of weight type $p$ is derived hereditary of type $\tilde{\tilde{\Delta}}$. 
Proof. The algebra $C$ is tilted of a hereditary algebra $H=k \tilde{\Delta}_{1}$, where $\tilde{\Delta}_{1}$ is a quiver with underlying graph $\tilde{\Delta}$. There is a derived equivalence $F: \mathrm{D}^{b}(\bmod -H) \rightarrow$ $\mathrm{D}^{b}(\bmod -C)$ sending the indecomposable projective $P_{i}$ corresponding to an extension vertex $i$ of $\tilde{\Delta}$ into the projective $C$-module $P_{\omega}$. Observe that $H\left[P_{i}\right]=k \tilde{\tilde{\Delta}}_{1}$ is a hereditary algebra where $\tilde{\tilde{\Delta}}_{1}$ is a quiver with underlying graph $\tilde{\tilde{\Delta}}$.

Let $\mathbb{X}=\mathbb{X}(p, \lambda)$ be a weighted projective line such that $\mathrm{D}^{b}(\bmod -C)=\mathrm{D}^{b}(\operatorname{coh}(\mathbb{X}))$. As an object in $\operatorname{coh}(\mathbb{X})$ the object $P_{\omega}$ has rank one (see [11]). Also by [11], there is an equivalence in $\mathrm{D}^{b}(\operatorname{coh}(\mathbb{X}))$ sending $P_{\omega}$ to any indecomposable preprojective $C$ module $N$. By [2], the one-point extension $C[N]$ is derived equivalent to $C\left[P_{\omega}\right]$ which is derived hereditary of type $\tilde{\tilde{\Delta}}$.

3.4. The converse of Proposition 3.3 also holds.

Proposition. Let $C=C(p, \lambda)$ be a canonical algebra and $P$ be an indecomposable projective $C$-module. The extended canonical algebra $A=C[P]$ is derived hereditary if and only if $C$ is tame domestic.

Proof. If $C$ is tame domestic, then $A$ is derived hereditary by (2.5). For the converse, consider the set of weight sequences $p=\left(p_{1}, p_{2}, \ldots, p_{t}\right)$ with $2 \leq p_{1} \leq p_{2} \leq \cdots \leq p_{t}$ with the domination order defined in (1.1).

The statement follows by induction on the domination order from the following two facts:

(a) a canonical tubular algebra $C$ is not derived hereditary;

(b) any wild weight sequence dominates a tubular one;

(c) if $M$ is an indecomposable $B$-module such that $B[M]$ is derived hereditary, then $B$ is derived hereditary.

(a): follows from the structure of derived categories of hereditary algebras, see [14]. (b) is clear.

(c): Assume $\mathrm{D}^{b}(\bmod -B[M])=\mathrm{D}^{b}(\bmod H)$ for a hereditary algebra $H$. By [12], $\mathrm{D}^{b}(\bmod -B)$ is equivalent to the right perpendicular category in $\mathrm{D}^{b}(\bmod -H)$ with respect to an exceptional object $E$, that is, $E$ is an indecomposable object satisfying $\operatorname{Ext}^{1}(E, E)=0$ and

$$
\mathrm{D}^{b}(\bmod -B)=E^{\perp}=\left\{X \in \mathrm{D}^{b}(\bmod -H): \operatorname{Hom}(E, X)=0=\operatorname{Ext}^{1}(E, X)\right\} .
$$


Without loss of generality we may assume that $E \in \bmod -H$. Then $\mathrm{D}^{b}(\bmod -B) \cong$ $\mathrm{D}^{b}\left(E^{\perp}\right)$, where now $E^{\perp}$ is formed in mod- $H$. Hence $E^{\perp}=\bmod -H^{\prime}$ for a hereditary algebra $H^{\prime}$.

3.5. Euler characteristic zero: the tubular case. Consider a canonical algebra $C=C(p, \lambda)$ with weight sequence $p=\left(p_{1}, \ldots, p_{t}\right)$, we shall assume that $2 \leq p_{1} \leq$ $p_{2} \leq \cdots \leq p_{t}$. The module category mod- $C$ accepts a separating tubular family $\mathcal{T}=\left(T_{\lambda}\right)_{\lambda \in \mathbb{P}_{1} k}$, where $T_{\lambda}$ is a homogeneous tube for all $\lambda$ with the exception of $t$ tubes $T_{\lambda_{1}}, \ldots, T_{\lambda_{t}}$ with $T_{\lambda_{i}}$ of rank $p_{i}(1 \leq i \leq t)$. See [34.

Let $\mathbb{X}=\mathbb{X}(p, \lambda)$ be the weighted projective line such that mod-C and $\operatorname{coh}(\mathbb{X})$ are derived equivalent. We fix an equivalence $\mathrm{D}^{b}(\bmod -C)=\mathrm{D}^{b}(\operatorname{coh}(\mathbb{X}))$. Let $S$ be a simple $C$-module in the mouth of the tube of rank $p_{t}$ and consider $S$ as an object in $\operatorname{coh}(\mathbb{X})$. The category $S^{\perp}$ right perpendicular to the object $S$ is the full subcategory of $\operatorname{coh}(\mathbb{X})$ consisting of all $\mathcal{F} \in \operatorname{coh}(\mathbb{X})$ satisfying

$$
\operatorname{Hom}_{\mathbb{X}}(S, \mathcal{F})=0=\operatorname{Ext}_{\mathbb{X}}^{1}(S, \mathcal{F}) .
$$

By [12], $S^{\perp}=\operatorname{coh}\left(\mathbb{X}^{\prime}\right)$ where $\mathbb{X}^{\prime}=\mathbb{X}\left(p^{\prime}, \lambda\right)$ is a weighted projective line with weight sequence $p^{\prime}=\left(p_{1}, p_{2}, \ldots, p_{t-1}, p_{t}-1\right)$. Moreover, if $0 \rightarrow \tau S \rightarrow U \rightarrow S \rightarrow 0$ is the almost split sequence in $\operatorname{coh}(\mathbb{X})$, then $U$ is a simple object in $S^{\perp}$ of $\tau^{\prime}$-period $p_{t}-1$, where $\tau^{\prime}=\tau_{\mathrm{D}^{b}\left(\operatorname{coh}\left(\mathbb{X}^{\prime}\right)\right)}$.

Proposition. Let $C=C(p, \lambda)$ be a canonical algebra of tubular type $p=\left(p_{1}, \ldots, p_{t}\right)$ and $A=C[P]$ be an extended canonical algebra. Then $A$ is derived canonical of type $\bar{p}=\left(p_{1}, \ldots, p_{t-1}, p_{t}+1\right)$.

Proof. By (2.4), we may choose $P$ to be the simple projective $C$-module. We shall show that $A$ is quasi-tilted of type $\bar{p}=\left(p_{1}, \ldots, p_{t-1}, p_{t}+1\right)$, see [15].

Let $\mathbb{X}$ be a weighted projective line with $\mathrm{D}^{b}(\operatorname{coh}(\mathbb{X}))=\mathrm{D}^{b}(\bmod C)$ and let $\overline{\mathbb{X}}$ denote a weighted projective line of type $\bar{p}$ such that $\operatorname{coh}(\mathbb{X})$ is the perpendicular category $S^{\perp}$ formed in $\operatorname{coh}(\overline{\mathbb{X}})$ for a simple $S$ from the tube of rank $p_{t}+1$. Let $U$ be the middle term of the almost split sequence $0 \rightarrow \tau S \rightarrow U \rightarrow S \rightarrow 0$ in $\operatorname{coh}(\overline{\mathbb{X}})$. Then $U$ is a simple in $S^{\perp}=\operatorname{coh}(\mathbb{X})$ belonging to the largest tube in $\operatorname{coh}(\mathbb{X})$.

By hypothesis, $\mathbb{X}$ has tubular type. By [22], there is a tilting object $\mathcal{T}$ in $\operatorname{coh}_{0}(\mathbb{X}) \vee$ $\operatorname{coh}_{+}(\mathbb{X})[1]$ such that End $(\mathcal{T})=C$, where $\operatorname{coh}_{0}(\mathbb{X})\left(\right.$ resp. $\left.\operatorname{coh}_{+}(\mathbb{X})\right)$ denotes the full 
subcategory of coh $(\mathbb{X})$ formed by the sheaves of rank 0 (resp. positive rank). Therefore, $\mathcal{T} \cup\{S\} \subset \operatorname{coh}_{0}(\overline{\mathbb{X}}) \vee \operatorname{coh}_{+}(\overline{\mathbb{X}})[1]$ is a tilting complex for $\overline{\mathbb{X}}$ whose endomorphism ring is isomorphic to $C[P]=A$.

3.6. Negative Euler characteristic: the wild case. For negative Euler characteristic the derived category of modules over an extended canonical algebra $C[P]$ relates to the study of the $\mathbb{Z}$-graded surface singularity $R$ associated with $C$ and the weighted projective line $\mathbb{X}$ associated to $C$. We refer to [11, 21, 12] for further details. The weighted projective line $\mathbb{X}=\mathbb{X}(p, \lambda)$ for a weight sequence $p=\left(p_{1}, \ldots, p_{t}\right)$ and a parameter sequence $\lambda=\left(\lambda_{3}, \ldots, \lambda_{t}\right)$ was introduced in [1] by means of the algebra

$$
S=S(p, \lambda):=k\left[x_{1}, \ldots, k_{t}\right] /\left(x_{i}^{p_{i}}=x_{2}^{p_{2}}-\lambda_{i} x_{1}^{p_{1}}\right), \quad i=3, \ldots, t .
$$

The algebra $S$ is naturally graded over the abelian group $\mathbb{L}(p)$ with generators $\vec{x}_{1}, \ldots, \vec{x}_{t}$ and relations $p_{1} \vec{x}_{1}=\cdots=p_{t} \vec{x}_{t}=: \vec{c}$ by giving each $x_{i}$ the degree $\vec{x}_{i}$. Here, $\vec{c}$ is called the canonical element of $\mathbb{L}(p)$. The group $\mathbb{L}(p)$ is isomorphic to the direct sum of the group $\mathbb{Z}$ of integers and some finite group. A quick way to arrive at the category $\operatorname{coh}(\mathbb{X})$ of coherent sheaves on $\mathbb{X}$ is by putting

$$
\operatorname{coh}(\mathbb{X})=\frac{\bmod ^{\mathbb{L}(p)}-S}{\bmod _{0}^{\mathbb{L}(p)}-S},
$$

where the quotient category on the right is formed in the sense of [10] and the categories $\bmod ^{\mathbb{L}(p)}-S$ (resp. $\bmod _{0}^{\mathbb{L}(p)}-S$ ) are the categories of finitely generated $\mathbb{L}(p)$-graded $S$-modules (resp. those of finite length).

The element $\vec{\omega}=(t-2) \vec{c}-\sum_{i=0}^{t} \vec{x}_{i}$ from $\mathbb{L}(p)$ is called the dualizing element. Its importance comes from the fact that Serre duality for coh $(\mathbb{X})$ holds in the form $\operatorname{DExt}^{1}(X, Y)=\operatorname{Hom}(Y, X(\vec{\omega}))$, where $X \mapsto X(\vec{\omega})$ is the self-equivalence of $\operatorname{coh}(\mathbb{X})$ induced by grading shift $M \mapsto M(\vec{\omega})$, given by $M(\vec{\omega})_{\vec{x}}=M_{\vec{\omega}+\vec{x}}$.

Assume that $\chi_{\mathbb{X}}<0$. Then the graded surface singularity $R=R(p, \lambda)$ attached to the weighted projective line $\mathbb{X}$ (or the canonical algebra $C$ ) with data $(p, \lambda)$ is defined as $R=\bigoplus_{n=0}^{\infty} R_{n}$, where $R_{n}=S_{n \vec{\omega}}$. It follows immediately that $R$ is a finitely generated, i.e. affine, $k$-algebra where each $R_{n}$ is finite dimensional over $k$ and, moreover, $R_{0}=k$ and $R_{1}=0$. The next theorem illustrates the role of $R$, and shows in particular that the algebra $R$ keeps all information on $\mathbb{X}$. For the proofs we refer to [21, 12]. 
Theorem. Assume $\chi_{\mathbb{X}}<0$. Then the following holds:

(a) The algebra $R=R(p, \lambda)$ is a positively $\mathbb{Z}$-graded isolated surface singularity which is graded Gorenstein of Gorenstein index -1 .

(b) There is a natural equivalence $\operatorname{coh}(\mathbb{X}) \rightarrow \bmod ^{\mathbb{Z}}-R / \bmod _{0}^{\mathbb{Z}}-R$, induced by restricting the grading from $\mathbb{L}(p)$ to $\mathbb{Z}=\mathbb{Z} \vec{\omega}$.

(c) For $k=\mathbb{C}$, the algebra $R(p, \lambda)$ is the positively $\mathbb{Z}$-graded algebra of automorphic forms on the (upper) complex half-plane $\mathbb{H}_{+}$with respect to the action of a Fuchsian group $G$ of the first kind of signature $\left(0 ; p_{1}, \ldots, p_{t}\right)$.

Concerning (a) we note that — restricting to the case of Krull dimension two - the Gorenstein index $d$ can be defined through the minimal graded injective resolution $0 \rightarrow R \rightarrow E^{0} \rightarrow E^{1} \rightarrow E^{2} \rightarrow 0$ of the $R$-module $R$, where the term $E^{2}$ is the graded injective hull of $k(d)$ and generally the grading shift $(n)$ is defined by $M(n)_{m}=$ $M_{n+m}$. In this situation, Serre duality holds for $\operatorname{coh}(\mathbb{X})$ in the form $\operatorname{DExt}^{1}(X, Y)=$ $\operatorname{Hom}(Y, X(-d))$, such that the Auslander-Reiten translation comes from the grading shift $X \mapsto X(-d)$.

Concerning (c) we remark that $G$ is the orbifold fundamental group of $\mathbb{X}$, having a presentation $\left\langle\sigma_{1}, \ldots, \sigma_{t} \mid \sigma_{1}^{p_{1}}=\ldots=\sigma_{t}^{p_{t}}=\sigma_{1} \cdots \sigma_{t}\right\rangle$ acting by covering transformations on the (branched) universal cover $\mathbb{H}_{+}$of $\mathbb{X}$. We refer to [26, 28] for the associated rings of automorphic forms.

3.7. For a variety $X$ Orlov investigated in [29] the triangulated category $\mathrm{D}_{\mathrm{Sg}}(X)$ of the singularities of $X$ defined as the quotient of the bounded derived category $\mathrm{D}^{b}(\operatorname{coh}(X))$ of coherent sheaves modulo the full subcategory of perfect complexes. If $X$ is affine with coordinate algebra $R$ this category $\mathrm{D}_{\mathrm{Sg}}(R)$ is just the quotient $\mathrm{D}^{b}(\bmod -R) / \mathrm{D}^{b}(\operatorname{proj}-R)$, where proj- $R$ is the category of finitely generated projective $R$-modules. In [30] Orlov further introduced a graded variant

$$
\mathrm{D}_{\mathrm{Sg}}^{\mathbb{Z}}(R)=\mathrm{D}^{b}\left(\bmod ^{\mathbb{Z}}-R\right) / \mathrm{D}^{b}\left(\operatorname{proj}^{\mathbb{Z}}-R\right)
$$

called the triangulated category of the graded singularity $R$ which will play a central role in this section.

Under the name stabilized derived category of $R$ the categories $\mathrm{D}_{\mathrm{Sg}}(R)$ were introduced by Buchweitz in [5]. His results easily extend to the graded case and yields for an $R$ that is graded Gorenstein an alternative description of $\mathrm{D}_{\mathrm{Sg}}^{\mathbb{Z}}(R)$ as the stable 
category of graded maximal Cohen-Macaulay modules ${\underline{\mathrm{MCM}^{\mathbb{Z}}}}^{\mathbb{R}}$. More precisely, he showed that the category $\mathrm{MCM}^{\mathbb{Z}}-R$ of maximal graded Cohen-Macaulay $R$-modules is a Frobenius-category, hence inducing - in Keller's terminology [18] — on the attached stable category $\underline{\mathrm{MCM}}^{\mathbb{Z}}-R$ of graded maximal Cohen-Macaulay modules modulo projectives, the structure of an algebraic triangulated category. For a related approach measuring the complexity of a singularity by a triangulated category we refer to Krause's account [19].

Let $R=\bigoplus_{n \geq 0} R_{n}, R_{n}=S_{n \vec{\omega}}$, be the positively $\mathbb{Z}$-graded Gorenstein singularity attached to the weighted projective line $\mathbb{X}$. It follows from [21, 5.6] that $R$ has Krull dimension two and Gorenstein index -1 . We fix some notation: Let $\mathcal{M}=$ $\mathrm{D}^{b}\left(\bmod ^{\mathbb{Z}}-R\right)$ and $\mathcal{M}_{+}=\mathrm{D}^{b}\left(\bmod ^{\mathbb{Z}_{+}}-R\right)$. Let $\mathcal{P}_{+}$be the triangulated subcategory of $\mathcal{M}_{+}$generated by all $R(-n), n \geq 0$ and $\mathcal{T}$ its left perpendicular category ${ }^{\perp} \mathcal{P}_{+}$formed in $\mathcal{M}_{+}$. Denote further by $\mathcal{S}_{+}$the triangulated subcategory of $\mathcal{M}_{+}$generated by all $k(-n), n \geq 0$ and $\mathcal{D}$ its right perpendicular category $\mathcal{S}_{+}^{\perp}$ formed in $\mathcal{M}_{+}$. Finally let $\mathcal{D}(-1)=\mathcal{S}_{+}(-1)^{\perp}$. Then [30, 2.5] implies the following proposition.

Proposition. Assume that $\chi_{\mathbb{X}}<0$ and let $R$ be the positively $\mathbb{Z}$-graded singularity attached to $\mathbb{X}$. Then the following holds:

(a) The natural functor $\mathcal{T} \hookrightarrow \mathcal{M} \stackrel{q}{\rightarrow} \mathrm{D}_{\mathrm{Sg}}(R)$, where $q$ is the quotient functor, is an equivalence of triangulated categories.

(b) The $R$-module $k$ is an exceptional object in $\mathcal{T}$ with ${ }^{\perp} k=\mathcal{D}(-1)$. Moreover, the category $\mathrm{D}^{b}(\operatorname{coh}(\mathbb{X}))$ is naturally equivalent to $\mathcal{D}(-1)$ under the functor $Y \mapsto$ $\left(\mathbf{R} \Gamma_{+}(Y)\right)(-1)$.

Proof. For the convenience of the reader we sketch the argument. Using that $R$ is Gorenstein of Gorenstein index -1, and invoking Gorenstein duality $\mathbf{R H o m}_{R}^{\bullet}(-, R)$ of $\mathcal{M}$ one sees that $T^{\perp} \subset \mathcal{D}(-1)^{\perp}$ and hence $\mathcal{D}(-1)$ is a full subcategory of $\mathcal{T}$. Further we see that ${ }^{\perp} k=\mathcal{D}(-1)$. It is well-known that

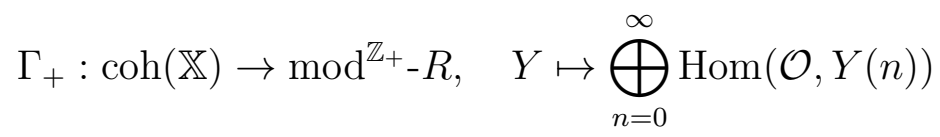

is a full embedding having sheafification, that is, the quotient functor $q_{+}: \bmod ^{\mathbb{Z}_{+}} R \rightarrow$ $\operatorname{coh}(\mathbb{X})$ as an exact left adjoint and such that composition $q \Gamma_{+}$is the identity functor on $\operatorname{coh}(\mathbb{X})$, compare [11, 1.8], [21, 5.7]. It follows that $\mathbf{R} \Gamma_{+}: \mathrm{D}^{b}(\operatorname{coh}(\mathbb{X})) \rightarrow \mathcal{M}_{+}$is a full embedding having $q_{+}: \mathcal{M}_{+} \rightarrow \mathcal{M}_{+} / \mathcal{S}_{+}$as a left adjoint, and $q_{+} \mathbf{R} \Gamma_{+}=1$. 
Since $R$ is positively graded with $R_{0}=k$, it follows that $k$ is exceptional in $\mathcal{M}$ and hence in $\mathcal{M}_{+}$. Invoking the minimal graded injective resolution $0 \rightarrow R \rightarrow E^{0} \rightarrow$ $E^{1} \rightarrow E^{2} \rightarrow 0$, where $E^{0}$ and $E^{1}$ are socle-free and $E^{2}$ is the graded injective hull of $k(-1)$, it follows that $k$ belongs to $\mathcal{T}$ and then also to $\mathcal{D}(-1)$. It is straightforward to check that $\mathcal{D}(-1)^{\perp}$ equals the triangulated subcategory $\langle k\rangle$ generated by $k$, and hence ${ }^{\perp} k=\mathcal{D}(-1)$ in $\mathcal{T}$.

3.8. Proof of Theorem 1. We are now in a position to clarify the structure of the category $\mathrm{D}_{\mathrm{Sg}^{-}}^{\mathbb{Z}}(R)$. The result was first observed by K. Saito and A. Takahashi (personal communication); it is not yet published, and uses the technique of matrix factorizations as in [17].

By Proposition 3.2 it suffices to show that the left adjoint $\ell: \mathcal{T} \rightarrow{ }^{\perp} k$ to the inclusion $j:{ }^{\perp} k \hookrightarrow \mathcal{T}$ maps $k$ to a line bundle in $\mathcal{D}(-1)=\mathrm{D}^{b}(\operatorname{coh}(\mathbb{X}))$ up to translation in $\mathcal{D}(-1)$. We put $A=\left(\mathbf{R} \Gamma_{+}(\mathcal{O}(-\vec{\omega}))\right)(-1)$ and construct a morphism $\gamma: k \rightarrow A[1]$ such that $\operatorname{Hom}(\gamma, Y): \operatorname{Hom}(A[1], Y) \rightarrow \operatorname{Hom}(k, Y)$ is an isomorphism for each $Y \in{ }^{\perp} k$ such that $\ell(k)=A[1]$.

The claim is proved in two steps. Put $R_{+}=\bigoplus_{n \geq 1} R_{n}$, then the exact sequence $0 \rightarrow R_{+} \rightarrow R \rightarrow k$ yields an exact triangle $R \rightarrow k \stackrel{\alpha}{\rightarrow} R_{+}[1]$ in $\mathcal{M}$, where $\operatorname{Hom}(\alpha, Y)$ is an isomorphism for each $Y \in{ }^{\perp} k$. Note for this that $R$ belongs to ${ }^{\perp} \mathcal{D}(-1)$.

For the next step it is useful to identify the derived category $\mathcal{M}_{+}$with the full subcategory of $\mathrm{D}^{b}\left(\mathrm{Mod}^{\mathbb{Z}_{+}}-R\right)$ consisting of all complexes with cohomology in $\bmod ^{\mathbb{Z}_{+}} R$. Here, $\operatorname{Mod}^{\mathbb{Z}_{+}} R$ denotes the category of all graded $R$-modules. Let $0 \rightarrow R(-1) \rightarrow$ $E^{0} \rightarrow E^{1} \rightarrow E^{2} \rightarrow 0$ be the minimal graded injective resolution of $R(1)$ such that $E^{2}$ equals the graded injective envelope of $k$. (This uses that $R$ has Gorenstein index -1 .) Sheafification yields the minimal injective resolution $0 \rightarrow \mathcal{O}(\vec{\omega}) \rightarrow \tilde{E}^{0} \rightarrow \tilde{E}^{1} \rightarrow 0$ of $\mathcal{O}(\vec{\omega})$. Accordingly $\mathbf{R} \Gamma_{+}(\mathcal{O}(\vec{\omega}))$ is given by the complex

$$
A: \quad \cdots \rightarrow 0 \rightarrow E_{+}^{0}(-1) \rightarrow E_{+}^{2}(-1) \rightarrow 0 \cdots, \text { where } E_{+}^{i}=\bigoplus_{n \geq 0} E_{n}^{i},
$$

whose cohomology is concentrated in degrees zero and one and given by

$$
\mathrm{H}^{0}(A)=R_{+}, \quad \mathrm{H}^{1}(A)=k(-1) .
$$

It follows the existence of an exact triangle

$$
k(-1)[-2] \rightarrow R_{+} \stackrel{\beta}{\rightarrow} A \rightarrow k(-1)[-1],
$$


in $\mathcal{M}_{+}$where, by construction, $A$ belongs to $\mathcal{D}(-1)$. For $Y$ from $\mathcal{D}(-1)$ we have, in particular, that $Y$ belongs to ${ }^{\perp} k(-1)$ implying that $\operatorname{Hom}(\beta, Y)$ is an isomorphism. To summarize: The morphism $\gamma=\left[k \stackrel{\alpha}{\rightarrow} R_{+}[1] \stackrel{\beta[1]}{\rightarrow} A[1]\right]$ yields isomorphisms $\operatorname{Hom}(\gamma, Y)$ for each $Y \in \mathcal{D}(-1)$. Hence $\ell(k)[-1]=A=\mathbf{R} \Gamma_{+}(\mathcal{O}(\vec{\omega}))(-1)$ is a line bundle, as claimed.

3.9. The Coxeter-Dynkin algebras of a singularity. In the theory of singularities the attached Coxeter-Dynkin diagrams, see for instance [8, 7], play an important role, in particular, since they establish a link to Lie theory.

Definition. Let $k$ be an algebraically closed field, and $R=R(p, \lambda)$ be the $\mathbb{Z}$-graded singularity attached to the weighted projective line $\mathbb{X}(p, \lambda)$.

(a) By the Coxeter-Dynkin algebra of hereditary type we mean the path algebra $D[p]$ of the hereditary star $\left[p_{1}, \ldots, p_{t}\right]$ having a unique sink.

(b) By the Coxeter-Dynkin algebra of canonical type we mean the algebra $D(p, \lambda)$ given in terms of the quiver

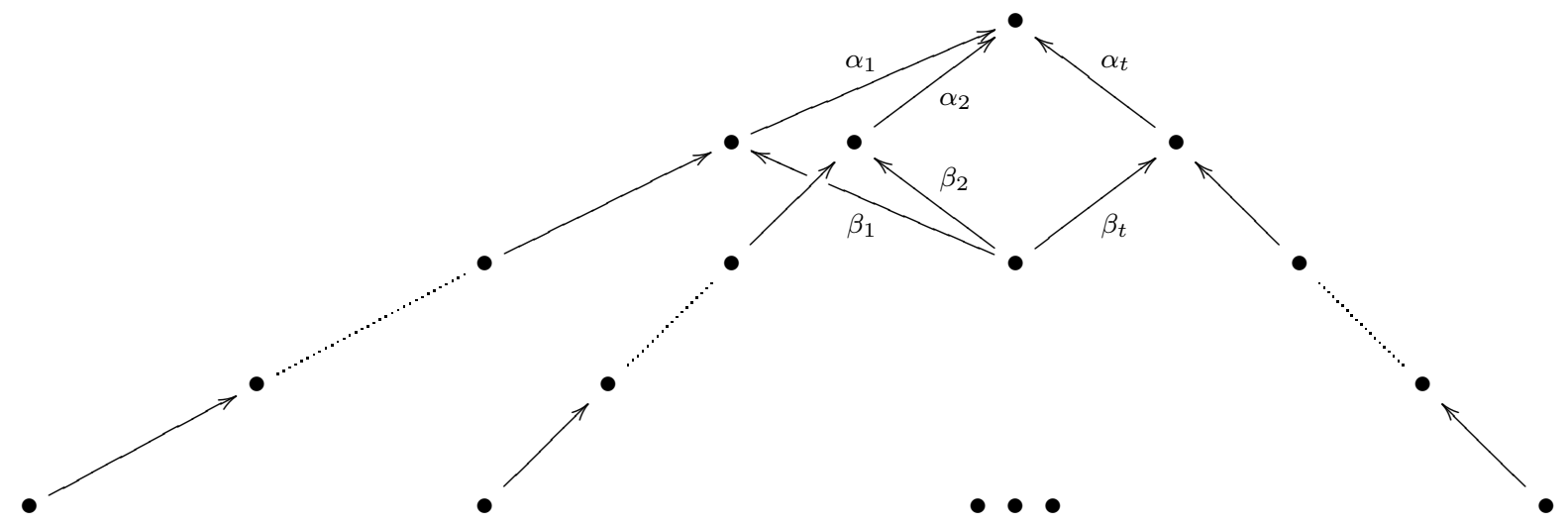

with the two relations $\sum_{i=2}^{t} \alpha_{i} \beta_{i}=0$ and $\alpha_{1} \beta_{1}=\sum_{i=3}^{t} \lambda_{i} \alpha_{i} \beta_{i}$.

(c) By the Coxeter-Dynkin algebra of extended-canonical type we mean the onepoint extension $\hat{D}(p, \lambda)$ of the Coxeter-Dynkin algebra $D(p, \lambda)$ of canonical type above, introducing a new arrow at the sink vertex and keeping the relations.

The link to singularity theory is given by the following well known result, compare [7, 8].

Theorem. For $k=\mathbb{C}$ the Coxeter-Dynkin diagram of the singularity $R(p, \lambda)$ is the underlying digraph of the Coxeter-Dynkin algebra

(a) of hereditary type, if $\chi_{\mathbb{X}}>0$, and then $\left[p_{1}, \ldots, p_{t}\right]$ is Dynkin. 
(b) of canonical type, if $\chi_{\mathbb{X}}=0$, and then $p$ is tubular.

(c) of extended canonical type, if $\chi_{\mathbb{X}}<0$.

Recall that the digraph of a finite dimensional algebra $A$ has the underlying graph of the quiver of $A$ as solid edges and the minimal number of relations between vertices $i$ and $j$ as dotted edges.

Proposition. Assume the number of weights $>1$ is at least two. Then the following holds:

(a) The Coxeter-Dynkin algebra $D(p, \lambda)$ of canonical type is derived equivalent to the canonical algebra $C(p, \lambda)$.

(b) The Coxeter-Dynkin algebra $\hat{D}(p, \lambda)$ of extended canonical type is derived equivalent to the extended canonical algebra $A=C[P]$, where $C=C(p, \lambda)$.

Proof. Let $\mathbb{X}$ be the weighted projective line with $t$ weighted points $x_{1}, \ldots, x_{t}$ of weight $p_{1}, \ldots, p_{t}$, respectively. For each $i=1, \ldots, t$ denote by $U_{i}$ the unique indecomposable sheaf of length $p_{i}-1$ concentrated in $x_{i}$ such there exists an epimorphism $\mathcal{O} \rightarrow U_{i}$ in $\operatorname{coh}(\mathbb{X})$, where $\mathcal{O}$ is the structure sheaf on $\mathbb{X}$. Moreover, let

$$
S_{i}=U_{i}^{(1)} \subset U_{i}^{(2)} \subset \cdots \subset U_{i}^{\left(p_{i}-1\right)}=U_{i}
$$

be the complete system of subobjects of $U_{i}$. It is straightforward to verify that the object

$$
T=\mathcal{O}(\vec{c}) \oplus \bigoplus_{i=1}^{t}\left(U_{i}^{(1)} \oplus U_{i}^{(2)} \oplus \cdots \oplus U_{i}^{\left(p_{i}-1\right)}\right) \oplus \mathcal{O}(-\vec{\omega})[1]
$$

is a tilting object in $\mathrm{D}^{b}(\operatorname{coh}(\mathbb{X}))$. Moreover, it is not difficult to see that the endomorphism ring of $T$ is isomorphic to the Coxeter-Dynkin algebra $D(p, \lambda)$.

This proves (a). Assertion (b) follows from (a) noting that $\mathcal{O}(-\vec{\omega})$ is a line bundle by applying [2] or arguing as in Proposition 3.2.

\subsection{The triangulated category of singularities for nonnegative Euler char-}

acteristic. In this paper we mainly concentrate on the case $\chi_{\mathbb{X}}<0$. To complete the picture we review the situation for $\chi_{\mathbb{X}} \geq 0$.

Assume that $k=\mathbb{C}$. For $\chi_{\mathbb{X}}>0$ the Dynkin diagram given by the weight type and the Coxeter-Dynkin diagram of the singularity $R=R(p, \lambda)$ agree. Moreover, it is shown in [17] that the category $\mathrm{D}_{\mathrm{Sg}}^{\mathbb{Z}}(R)$ is equivalent to the bounded derived category $\mathrm{D}^{b}(k \Delta)$ of the path algebra of a quiver of the same Dynkin type. 
Next we deal with the case $\chi_{\mathbb{X}}=0$. First, there is no $\mathbb{Z}$-graded Gorenstein algebra $R$ such that $\mathcal{C}=\bmod ^{\mathbb{Z}}-R / \bmod _{0}^{\mathbb{Z}}-R$ is equivalent to $\operatorname{coh}(\mathbb{X})$. Assume, indeed, that such an algebra $R$ would exist. Let $d$ denote its Gorenstein index. Since the Auslander-Reiten translation $\tau(X)=X(-d)$ has finite order 2, 3, 4 or 6 for any weighted projective line $\mathbb{X}$ of tubular type, it follows that $d=0$, hence $\tau$ is the identity, contradiction.

Hence for $\chi_{\mathbb{X}}=0$ it is more natural to investigate the $\mathbb{L}(p)$-graded singularity $S=S(p, \lambda)$ and its triangulated category of singularities $\mathrm{D}_{\mathrm{Sg}}^{\mathbb{L}(p)}(S)$ which in the tubular case is equivalent to $\mathrm{D}^{b}(\operatorname{coh}(\mathbb{X}))$ due to recent work of Ueda [35].

\section{The Coxeter polynomial of an extended canonical algebra}

4.1. Let $A$ be a finite dimensional $k$-algebra of finite global dimension. The Coxeter transformation $\varphi_{A}: K_{0}(A) \rightarrow K_{0}(A)$ is the automorphism induced by the AuslanderReiten translation $\tau_{\mathrm{D}^{b}(\bmod A)}: \mathrm{D}^{b}(\bmod -A) \rightarrow \mathrm{D}^{b}(\bmod -A)$. We shall consider $\varphi_{A}$ as a $n \times n$ integral matrix where $n$ is the rank of $K_{0}(A)$. ¿From the Introduction, we recall that $f_{A}(T)=\operatorname{det}\left(T \mathrm{id}-\varphi_{A}\right)$ is called the Coxeter polynomial of $A$.

For a one-point extension $A=B[P]$ of an algebra $B$ by an indecomposable projective a simple calculation shows (see [24, 34]):

$$
f_{A}(T)=(1+T) f_{B}(T)-T f_{C}(T) .
$$

Of particular interest is the hereditary case where any algebra can be constructed by repeated one-point extensions using indecomposable projective modules. In fact, for a star $H$ of type $\left[p_{1}, \ldots, p_{t}\right]$ the above formula yields:

$$
f_{\left[p_{1}, \ldots, p_{t}\right]}(T):=f_{H}(T)=\left[(T+1)-T \sum_{i=1}^{t} \frac{v_{p_{i}-1}}{v_{p_{i}}}\right] \prod_{i=1}^{t} v_{p_{i}}
$$

where we set $v_{n}(T)=\frac{T^{n}-1}{T-1}=\sum_{i=0}^{n-1} T^{i}$.

For the canonical algebra $C=C(p, \lambda)$ of type $p=\left(p_{1}, \ldots, p_{t}\right)$, we get

$$
f_{\left(p_{1}, \ldots, p_{t}\right)}(T):=f_{C}(T)=(T-1)^{2} \prod_{i=1}^{t} v_{p_{i}}(T) .
$$

In particular, all the eigenvalues of $\varphi_{C}$ lie on the unit circle $\mathbb{S}^{1}$. For these calculations we refer the reader to [23]. 
Lemma [24]. An extended canonical algebra $A=C[P]$ where $C=C(p, \lambda)$ with $p=\left(p_{1}, \ldots, p_{t}\right)$ has Coxeter polynomial:

$$
\hat{f}_{\left(p_{1}, \ldots, p_{t}\right)}(T):=f_{A}(T)=(T+1)(T-1)^{2} \prod_{i=1}^{t} v_{p_{i}}(T)-T f_{\left[p_{1}, \ldots, p_{t}\right]}(T)
$$

4.2. For later use we recall some facts on cyclotomic polynomials.

The $n$-cyclotomic polynomial $\phi_{n}(T)$ is inductively defined by the formula

$$
T^{n}-1=\prod_{d \mid n} \phi_{d}(\mathcal{T})
$$

Recall that the Möbius function is defined as follows:

$$
\mu(n)= \begin{cases}0 & \text { if } n \text { is divisible by a square } \\ (-1)^{r} & \text { if } n=p_{1}, \ldots p_{r} \text { is a factorization into distinct primes. }\end{cases}
$$

A more explicit expression for the cyclotomic polynomials is given by:

Lemma. For each $n \geq 2$, we have

$$
\phi_{n}(T)=\prod_{\substack{1 \leq d<n \\ d \mid n}} v_{n / d}(T)^{\mu(d)}
$$

4.3. Following [24] we say that a polynomial $p(T) \in \mathbb{Z}[T]$ is represented by $q(T) \in$ $\mathbb{Z}[T]$ if

$$
p\left(T^{2}\right)=q^{*}(T):=T^{\operatorname{deg} q} q\left(T+T^{-1}\right) .
$$

The interest in the representability of polynomials is due to the relation between the set of roots of $p(T)$ and $q(T)$ whenever $p\left(T^{2}\right)=q^{*}(T)$. Indeed, in that case $\operatorname{Root} p(T) \subset \mathbb{S}^{1}\left(\operatorname{resp} . \mathbb{S}^{1} \backslash\{1\}\right)$ if and only if $\operatorname{Root} q(T) \subset[-2,2]$ (resp. $(-2,2)$ ). In [24] is shown that the Coxeter polynomial $\hat{f}_{\left(p_{1}, \ldots, p_{t}\right)}(T)$ of an extended canonical algebra of type $\left(p_{1}, \ldots, p_{t}\right)$ is represented by

$$
q_{\left(p_{1}, \ldots, p_{t}\right)}(T)=T\left(T^{2}-1\right) \prod_{i=1}^{t} v_{p_{i}}(T)-\chi_{\left[p_{1}, \ldots, p_{t}\right]}(T),
$$

where $\chi_{\left[p_{1}, \ldots, p_{t}\right]}(T)$ is the characteristic polynomial of the adjacency matrix of the star graph of type $\left[p_{1}, \ldots, p_{t}\right]$.

Using the above expressions the following was recently shown by the authors:

Theorem [24]. Let $A=C[P]$ be an extended canonical algebra of type $\left(p_{1}, \ldots, p_{t-1}, p_{t}+\right.$ $1)$ and $A^{\prime}$ be an extended canonical algebra of type $\left(p_{1}, \ldots, p_{t}\right)$. Then the following holds: 
(a) $\varphi_{A}$ accepts at most 4 eigenvalues outside $\mathbb{S}^{1}$

(b) If Root $f_{A} \subset \mathbb{S}^{1}$, then also Root $f_{A^{\prime}} \subset \mathbb{S}^{1}$

Sketch of proof: The polynomials $q_{\left(p_{1}, \ldots, p_{t}\right)}(T)$ satisfy a Chebysheff recursion formula as follows:

$$
q_{\left(p_{1}, \ldots, p_{t}+1\right)}(T)=T q_{\left(p_{1}, \ldots, p_{t}\right)}(T)-q_{\left(p_{1}, \ldots, p_{t}-1\right)}(T) .
$$

A version of Sturm's Theorem applies to assure that for any real interval $[\alpha, \beta]$, if $q_{\left(p_{1}, \ldots, p_{t}+1\right)}(T)$ has roots $\lambda_{1} \leq \cdots \leq \lambda_{s}$ in $[\alpha, \beta]$, then $q_{\left(p_{1}, \ldots, p_{t}\right)}(T)$ has roots $\lambda_{1}^{\prime} \leq \cdots \leq$ $\lambda_{s-1}^{\prime}$ in $[\alpha, \beta]$ satisfying

$$
\lambda_{1} \leq \lambda_{1}^{\prime} \leq \lambda_{2} \leq \lambda_{2}^{\prime} \leq \cdots \leq \lambda_{s-1} \leq \lambda_{s-1}^{\prime} \leq \lambda_{s}
$$

(a) and (b) follow easily from these facts.

4.4. According to (4.3), to prove Theorem 2 we need to calculate the minimal weight sequences $p$ such that $\hat{f}_{p}(T)$ is not contained in $\mathbb{S}^{1}$. This is done by systematically computing the roots of Coxeter polynomials of extended canonical algebras.

Recall that the spectral radius of the Coxeter transformation $\varphi_{A}$ is by definition $\rho_{A}\left(\varphi_{A}\right)=\max \left\{|\lambda|: \lambda \in \operatorname{Root} f_{A}(T)\right\}$. In the following list $A$ is an extended canonical algebra of weight type $p$. The invariant called Dynkin index is explained in section 5 , 


\begin{tabular}{|c|c|c|c|c|}
\hline & $\begin{array}{c}\text { Weight } \\
\left(p_{1}, \ldots, p_{t}\right)\end{array}$ & Irreducible factorization of $f_{A}(T)$ & $\rho\left(\varphi_{A}\right)$ & \begin{tabular}{|c} 
Dynkin \\
index
\end{tabular} \\
\hline \multirow[t]{9}{*}{$t=3$} & $(2,3,11)$ & $1+T-T^{3}-T^{4}+T^{6}+T^{7}+T^{9}+T^{10}-T^{12}-T^{13}+T^{15}+T^{16}$ & 1.1064 & 6 \\
\hline & $(2,4,9)$ & $\phi_{2} \phi_{5}\left(T^{10}-T^{9}+T^{5}+T+1\right)$ & 1.1329 & 4 \\
\hline & $(2,5,8)$ & $1+T+T^{4}+T^{5}+T^{6}+2 T^{8}+T^{9}+T^{10}+T^{11}+T^{14}+T^{15}$ & 1.1574 & 4 \\
\hline & $(2,6,7)$ & $1+T+T^{4}+2 T^{5}+2 T^{6}+T^{7}+T^{8}+2 T^{9}+2 T^{10}+T^{11}+T^{14}+T^{15}$ & 1.1669 & 4 \\
\hline & $(3,3,8)$ & $1+T+T^{2}+T^{5}+2 T^{6}+3 T^{7}+2 T^{8}+T^{9}+T^{12}+T^{13}+T^{14}$ & 1.1498 & 3 \\
\hline & $(3,4,7)$ & $1+T+T^{2}+T^{3}+T^{4}+2 T^{5}+3 T^{6}+3 T^{7}+3 T^{8}+2 T^{9}+T^{10}+T^{11}+T^{12}+T^{13}+T^{14}$ & 1.1847 & 3 \\
\hline & $(3,5,6)$ & $\phi_{3}\left(T^{12}+T^{9}+T^{8}+T^{7}+T^{6}+T^{5}+T^{4}+T^{3}+1\right)$ & 1.1966 & 3 \\
\hline & $(4,4,6)$ & $\phi_{2} \phi_{4}\left(T^{10}-T^{9}+T^{8}+T^{6}+T^{4}+T^{2}-T+1\right)$ & 1.2715 & 3 \\
\hline & $(4,5,5)$ & $\phi_{5}\left(T^{10}+T^{7}+T^{6}+T^{5}+T^{4}+T^{3}+1\right)$ & 1.2277 & 3 \\
\hline \multirow[t]{4}{*}{$t=4$} & $(2,2,2,7)$ & $\phi_{2}^{2}\left(T^{10}+T^{6}+T^{5}+T^{4}+1\right)$ & 1.1670 & 2 \\
\hline & $(2,2,3,6)$ & $\phi_{2}^{2} \phi_{3}\left(T^{8}-T^{7}+T^{6}+T^{4}+T^{2}-T+1\right)$ & 1.2196 & 2 \\
\hline & $(2,3,3,4)$ & $\phi_{2} \phi_{4}\left(T^{8}+T^{6}+T^{5}+2 T^{4}+T^{3}+T^{2}+1\right)$ & 1.2874 & 2 \\
\hline & $(3,3,3,4)$ & $\phi_{3}^{2}\left(T^{8}+T^{6}+2 T^{5}+2 T^{3}+T^{2}+1\right)$ & 1.3307 & 2 \\
\hline \multirow[t]{4}{*}{$t=5$} & & & & 2 \\
\hline & $(2,2,2,2,5)$ & $\phi_{2}^{3}\left(T^{8}+T^{6}+T^{3}+2 T^{4}+T^{3}+T^{2}+1\right)$ & 1.2874 & 2 \\
\hline & $(2,2,2,3,4)$ & $\phi_{2}^{3}\left(T^{8}+2 T^{6}+T^{5}+3 T^{4}+2 T^{2}+1\right)$ & 1.3351 & 2 \\
\hline & $(2,2,3,3,3)$ & $\phi_{2} \phi_{3}^{2}\left(T^{6}+T^{4}+2 T^{3}+T^{2}+1\right)$ & 1.3765 & 2 \\
\hline \multirow[t]{2}{*}{$t=6$} & & & & 2 \\
\hline & $(2,2,2,2,2,3)$ & $\phi_{2}^{4}\left(T^{6}+2 T^{4}+T^{3}+2 T^{2}+1\right)$ & 1.3395 & 2 \\
\hline \multirow[t]{2}{*}{$t=7$} & & & & 2 \\
\hline & $(2,2,2,2,2,2,2)$ & $\phi_{2}^{6}\left(T^{4}-T^{3}+3 T^{2}-T+1\right)$ & 1.5392 & 2 \\
\hline
\end{tabular}

Table 1. Critical weight sequences.

4.5. To complete the proof of Theorem 2 we shall show that any weight sequence $p^{\prime}<p$ with $p$ in Table 1 has all its roots on $\mathbb{S}^{1}$. This is computed in the following Table 2.

As above $A=C[P]$ is an extended canonical algebra of weight type $p=\left(p_{1}, \ldots, p_{t}\right)$. In Table 2 , the marks $\bullet$ and $\square$ refer to the case $k=\mathbb{C}$ : those weight sequences marked 
by • or $\square$ correspond to algebras $R(p, \lambda)$ associated to hypersurface singularities, in those cases $R(p, \lambda)$ is formally 3-generated. The marks - correspond to Arnold's 14 exceptional unimodal singularities in the theory or singularities of differentiable maps [1]. Among those weight sequences $p=\left(p_{1}, p_{2}, p_{3}\right)$ (that is $t=3$ ), Arnold's singularities are exactly those rings of automorphic forms having three generators [37. 


\begin{tabular}{|c|c|c|c|c|}
\hline \multicolumn{2}{|c|}{ Weight sequence } & Factorization of $f_{A}(T)$ & Poincaré series & Period of $\varphi_{A}$ \\
\hline • & $(2,3,7)$ & $\phi_{42}$ & $(6,14,21)(42)$ & 42 \\
\hline • & $(2,3,8)$ & $\phi_{2} \cdot \phi_{10} \cdot \phi_{30}$ & $(6,8,15)(30)$ & 30 \\
\hline - & $(2,3,9)$ & $\phi_{3} \cdot \phi_{12} \cdot \phi_{24}$ & $(6,8,9)(24)$ & 24 \\
\hline & $(2,3,10)$ & $\phi_{2} \cdot \phi_{16} \cdot \phi_{18}$ & $(6,8,9,10)(16,18)$ & 72 \\
\hline \multicolumn{5}{|c|}{----------------------------------------} \\
\hline • & $(2,4,5)$ & $\phi_{2} \cdot \phi_{6} \cdot \phi_{30}$ & $(4,10,15)(30)$ & 30 \\
\hline • & $(2,4,6)$ & $\phi_{2}^{2} \cdot \phi_{22}$ & $(4,6,11)(22)$ & 22 \\
\hline \multirow[t]{2}{*}{ • } & $(2,4,7)$ & $\phi_{2} \cdot \phi_{9} \cdot \phi_{18}$ & $(4,6,7)(18)$ & 18 \\
\hline & $(2,4,8)$ & $\phi_{2}^{2} \cdot \phi_{4} \cdot \phi_{12} \cdot \phi_{14}$ & $(4,6,7,8)(12,14)$ & 84 \\
\hline \multicolumn{5}{|c|}{----------------------------------------} \\
\hline • & $(2,5,5)$ & $\phi_{5} \cdot \phi_{20}$ & $(4,5,10)(20)$ & 20 \\
\hline \multirow[t]{3}{*}{ • } & $(2,5,6)$ & $\phi_{2} \cdot \phi_{8} \cdot \phi_{16}$ & $(4,5,6)(16)$ & 16 \\
\hline & $(2,5,7)$ & $\phi_{11} \cdot \phi_{12}$ & $(4,5,6,7)(11,12)$ & 132 \\
\hline & $(2,6,6)$ & $\phi_{2}^{2} \cdot \phi_{3} \cdot \phi_{6} \cdot \phi_{10} \cdot \phi_{12}$ & $(4,5,6,6)(10,12)$ & 60 \\
\hline \multicolumn{5}{|c|}{------------------------------------} \\
\hline • & $(3,3,4)$ & $\phi_{3} \cdot \phi_{24}$ & $(3,8,12)(24)$ & 24 \\
\hline • & $(3,3,5)$ & $\phi_{2} \cdot \phi_{3} \cdot \phi_{6} \cdot \phi_{18}$ & $(3,5,9)(18)$ & 18 \\
\hline \multirow[t]{2}{*}{ • } & $(3,3,6)$ & $\phi_{3}^{2} \cdot \phi_{15}$ & $(3,5,6)(15)$ & 15 \\
\hline & $(3,3,7)$ & $\phi_{2} \cdot \phi_{3} \cdot \phi_{4} \cdot \phi_{10} \cdot \phi_{12}$ & $(3,5,6,7)(10,12)$ & 60 \\
\hline • & $(3,4,4)$ & $\phi_{2} \cdot \phi_{4} \cdot \phi_{16}$ & $(3,4,8)(16)$ & 16 \\
\hline \multirow[t]{3}{*}{ • } & $(3,4,5)$ & $\phi_{13}$ & $(3,4,5)(13)$ & 13 \\
\hline & $(3,4,6)$ & $\phi_{2} \cdot \phi_{3} \cdot \phi_{9} \cdot \phi_{10}$ & $(3,4,5,6)(9,10)$ & 90 \\
\hline & $(3,5,5)$ & $\phi_{2} \cdot \phi_{5} \cdot \phi_{8} \cdot \phi_{10}$ & $(3,4,5,5)(8,10)$ & 40 \\
\hline \multirow[t]{2}{*}{ • } & $(4,4,4)$ & $\phi_{2}^{2} \cdot \phi_{4}^{2} \cdot \phi_{6} \cdot \phi_{12}$ & $(3,4,4)(12)$ & 12 \\
\hline & $(4,4,5)$ & $\phi_{2} \cdot \phi_{4} \cdot \phi_{8} \cdot \phi_{9}$ & $(3,4,4,5)(8,9)$ & 72 \\
\hline \multicolumn{5}{|c|}{--------------------------------------} \\
\hline 口 & $(2,2,2,3)$ & $\phi_{2}^{2} \cdot \phi_{18}$ & $(2,6,9)(18)$ & 18 \\
\hline 口 & $(2,2,2,4)$ & $\phi_{2}^{2} \cdot \phi_{14}$ & $(2,4,7)(14)$ & 14 \\
\hline \multirow[t]{2}{*}{ 口 } & $(2,2,2,5)$ & $\phi_{2}^{2} \cdot \phi_{3} \cdot \phi_{6} \cdot \phi_{12}$ & $(2,4,5)(12)$ & 12 \\
\hline & $(2,2,2,6)$ & $\phi_{2}^{2} \cdot \phi_{8} \cdot \phi_{10}$ & $(2,4,5,6)(8,10)$ & 40 \\
\hline$\square$ & $(2,2,3,3)$ & $\phi_{2} \cdot \phi_{3} \cdot \phi_{4} \cdot \phi_{12}$ & $(2,3,6)(12)$ & 12 \\
\hline \multirow[t]{3}{*}{ 口 } & $(2,2,3,4)$ & $\phi_{2}^{2} \cdot \phi_{5} \cdot \phi_{10}$ & $(2,3,4)(10)$ & 10 \\
\hline & $(2,2,3,5)$ & $\phi_{2} \cdot \phi_{7} \cdot \phi_{8}$ & $(2,3,4,5)(7,8)$ & 56 \\
\hline & $(2,2,4,4)$ & $\phi_{2}^{2} \cdot \phi_{4} \cdot \phi_{6} \cdot \phi_{8}$ & $(2,3,4,4)(6,8)$ & 24 \\
\hline \multirow[t]{3}{*}{$\square$} & $(2,3,3,3)$ & $\phi_{3}^{2} \cdot \phi_{9}$ & $(2,3,3)(9)$ & 9 \\
\hline & $(2,3,3,4)$ & $\phi_{2} \cdot \phi_{3} \cdot \phi_{6} \cdot \phi_{7}$ & $(2,3,3,4)(6,7)$ & 42 \\
\hline & $(3,3,3,3)$ & $\phi_{2} \cdot \phi_{3}^{3} \cdot \phi_{6}^{2}$ & $(2,3,3,3)(6,6)$ & $\infty$ \\
\hline 口 & $(2,2,2,2,2)$ & $\phi_{2}^{4} \cdot \phi_{10}$ & $(2,2,5)(10)$ & 10 \\
\hline \multirow[t]{4}{*}{ 口 } & $(2,2,2,2,3)$ & $\phi_{2}^{3} \cdot \phi_{4} \cdot \phi_{8}$ & $(2,2,3)(8)$ & 8 \\
\hline & $(2,2,2,2,4)$ & $\phi_{2}^{2} \cdot \phi_{3} \cdot \phi_{6}^{2}$ & $(2,2,3,4)(6,6)$ & $\infty$ \\
\hline & $(2,2,2,3,3)$ & $\phi_{2}^{2} \cdot \phi_{3} \cdot \phi_{5} \cdot \phi_{6}$ & $(2,2,3,3)(5,6)$ & 30 \\
\hline & $(2,2,2,2,2,2)$ & $\phi_{2}^{5} \cdot \phi_{4} \cdot \phi_{6}$ & $(2,2,2,3)(4,6)$ & 12 \\
\hline
\end{tabular}

Table 2. Weights $p$ with $\rho\left(\varphi_{A}\right)=1$. 
4.6. Proof of Theorem 2, (a) follows from (4.4) and (4.5) using Theorem (4.3). Part (b) is shown in [24], see (4.3).

\section{The Poincaré series of an extended canonical algebra}

5.1. Let $C=C(p, \lambda)$ be a wild canonical algebra with weight sequence $p=\left(p_{1}, \ldots, p_{t}\right)$. Let $P$ be an indecomposable projective $C$-module. We define the Poincaré series $\hat{P}_{C}=\hat{P}\left(p_{1}, \ldots, p_{t}\right) \in \mathbb{Z}[[T]]$ by

$$
\hat{P}_{C}(T)=\sum_{n=0}^{\infty}\left\langle[P], \varphi_{C}^{n}[P]\right\rangle_{C} T^{n} .
$$

Recall that $\varphi_{C}^{n}[P]=\left[\tau_{\mathrm{D}^{b}(\bmod -C)}^{n} P\right]$ in $K_{0}\left(\mathrm{D}^{b}(\bmod -C)\right)=K_{0}(C)$. Moreover, observe that $T+\hat{P}_{C}(T)$ is the Hilbert-Poincaré series $P_{C}(T)$, as defined in the Introduction, for the graded algebra

$$
R(p, \lambda)=\bigoplus_{n=0}^{\infty} \operatorname{Hom}\left(L, \tau_{\mathbb{X}}^{n} L\right)
$$

where $\mathbb{X}=\mathbb{X}(p, \lambda)$ is a weighted projective line, $\tau_{\mathbb{X}}$ is the Auslander-Reiten translation in $\operatorname{coh}(\mathbb{X})$ and $L$ is any rank one bundle, see [23] and [25]. In particular, $\hat{P}_{C}(T)$ does not depend on the choice of $P$.

Proposition. With the notation above, let $A=C[P]$ be an extended canonical algebra. Then

(a) [25, Cor 3.6]: $f_{A}(T)=P_{C}(T) f_{C}(T)$

(b) [23, Th. 8.6]: $P_{C}(T)=1+T-T \frac{f_{\left[p_{1}, \ldots, p_{t}\right]}(T)}{f_{\left(p_{1}, \ldots, p_{t}\right)}(T)}$

(c) [23, Prop. 4.3]: $P_{C}(T)=T+\frac{1}{1-T}+(t-2) \frac{T}{(1-T)^{2}}-\sum_{i=1}^{t} \frac{T}{(1-T)\left(1-T^{p_{i}}\right)}$.

5.2. We recall from [23] the following concepts.

Definition [23]. Assume $p=\left(p_{1}, \ldots, p_{t}\right)$ is a weight sequence of wild type. The Dynkin label of $p$ is the Dynkin diagram of one of the extended Dynkin graphs $[2,2,2,2]$, $[3,3,3][2,4,4]$ or $[2,3,6]$ specified as follows:

(a) if $t \geq 4$, then the label is of type $[2,2,2,2]$

(b) if $t=3$, then the label is of type $[a, b, c]$ if $[a, b, c] \leq\left[p_{1}, p_{2}, p_{3}\right]$ and $a+b+c$ is minimal.

We say that $p$ has Dynkin index 2, 3, 4 or 6 if its Dynkin label is $[2,2,2,2],[3,3,3]$, $[2,4,4]$ or $[2,3,6]$ respectively. 
Consider the graded algebra $R=R(p, \lambda)$. In [23] the support monoid $M(p)$ was introduced as the set of those $n \in \mathbb{N}$ with $R_{n} \neq 0$. Clearly, $M(p)$ is an additive semigroup in $\mathbb{N}$ generating $\mathbb{Z}$ as a group.

Proposition [23]. The support monoid $M(p)$ is finitely generated with at most 6 generators. The smallest element in $M(p)$ is the Dynkin index of $p$.

5.3. In a preliminary version of [23], the authors displayed the list of all possible support monoids $M(p)$. This list of 22 semigroups is essential for the proofs of Theorems 3 and 4 and we reproduce it below. For a weight sequence $p$ the largest integer $n$ such that $n$ does not belong to $M(p)$ is called the Frobenius number of $M(p)$ and it is denoted by $\alpha(p)$. Of particular interest is the fact that $p \leq q$ implies $M(p) \subset M(q)$. 


\begin{tabular}{|c|c|c|}
\hline Weight type $p$ & Frobenius number $\alpha(p)$ & generators of $\mathbb{M}(p)$ \\
\hline $\begin{array}{c}\mathbf{( 2 , 3}, \mathbf{7}) \\
(2,3,8) \\
(2,3,9) \\
(2,3,10) \\
(2,3,11) \\
(2,3,12) \\
(2,3,13) \\
(2,3, \infty)\end{array}$ & $\begin{array}{c}43 \\
25 \\
19 \\
13 \\
13 \\
13 \\
7 \\
7 \\
\end{array}$ & $\begin{array}{c}\{6,14,21\} \\
\{6,8,15\} \\
\{6,8,9\} \\
\{6,8,9,10\} \\
\{6,8,9,10,11\} \\
\{6,8,9,10,11\} \\
\{6,8,9,10,11,13\} \\
\{6,8,9,10,11,13\}\end{array}$ \\
\hline $\begin{array}{c}(\mathbf{2}, \mathbf{4}, \mathbf{5}) \\
(2,4,6) \\
(2,4,7) \\
(2,4,8) \\
(2,4,9) \\
(2,4, \infty)\end{array}$ & $\begin{array}{l}21 \\
13 \\
9 \\
9 \\
5 \\
5\end{array}$ & $\begin{array}{c}\{4,10,15\} \\
\{4,6,11\} \\
\{4,6,7\} \\
\{4,6,7\} \\
\{4,6,7,9\} \\
\{4,6,7,9\}\end{array}$ \\
\hline $\begin{array}{c}(2,5,5) \\
(2,5,6) \\
(2,5,7) \\
(2,5, \infty)\end{array}$ & $\begin{array}{c}11 \\
7 \\
3 \\
3\end{array}$ & $\begin{array}{c}\{4,5\} \\
\{4,5,6\} \\
\{4,5,6,7\} \\
\{4,5,6,7\}\end{array}$ \\
\hline $\begin{array}{l}(2,6,6) \\
(2,6,7) \\
(2,6, \infty)\end{array}$ & $\begin{array}{l}7 \\
3 \\
3 \\
\end{array}$ & $\begin{array}{c}\{4,5,6\} \\
\{4,5,6,7\} \\
\{4,5,6,7\}\end{array}$ \\
\hline$(2, \infty, \infty)$ & 3 & $\{4,5,6,7\}$ \\
\hline $\begin{array}{c}\mathbf{3}, \mathbf{3}, \mathbf{4}) \\
(3,3,5) \\
(3,3,6) \\
(3,3,7) \\
(3,3, \infty)\end{array}$ & $\begin{array}{c}13 \\
7 \\
7 \\
4 \\
3 \\
\end{array}$ & $\begin{array}{c}\{3,8\} \\
\{3,5\} \\
\{3,5\} \\
\{3,5,7\} \\
\{3,5,7\}\end{array}$ \\
\hline $\begin{array}{l}(3,3,4) \\
(3,3,5)\end{array}$ & $\begin{array}{l}5 \\
2\end{array}$ & $\begin{array}{c}\{3,4\} \\
\{3,4,5\}\end{array}$ \\
\hline $\begin{array}{c}(4,4,4) \\
(4,4,5) \\
(\infty, \infty, \infty)\end{array}$ & $\begin{array}{l}5 \\
2 \\
2\end{array}$ & $\begin{array}{c}\{3,4\} \\
\{3,4,5\} \\
\{3,4,5\}\end{array}$ \\
\hline $\begin{array}{c}(\mathbf{2}, \mathbf{2}, \mathbf{2}, \mathbf{3}) \\
(2,2,2,4) \\
(2,2,2,5) \\
(2,2,2, \infty)\end{array}$ & $\begin{array}{l}7 \\
5 \\
3 \\
3 \\
\end{array}$ & $\begin{array}{l}\{2,9\} \\
\{2,7\} \\
\{2,5\} \\
\{2,5\}\end{array}$ \\
\hline $\begin{array}{c}(2,2,3,3) \\
(2,2,3, \infty)\end{array}$ & $\begin{array}{l}1 \\
1\end{array}$ & $\begin{array}{l}\{2,3\} \\
\{2,3\}\end{array}$ \\
\hline$(\infty, \infty, \infty, \infty)$ & 1 & $\{2,3\}$ \\
\hline $\begin{array}{c}\mathbf{( 2 , 2}, \mathbf{2}, \mathbf{2}, \mathbf{2}) \\
(2,2,2,2,3) \\
(2,2,2,2, \infty)\end{array}$ & $\begin{array}{l}3 \\
1 \\
1\end{array}$ & $\begin{array}{l}\{2,5\} \\
\{2,3\} \\
\{2,3\}\end{array}$ \\
\hline $\begin{array}{c}(2,2,2,2,2,2) \\
(\infty, \infty, \ldots, \infty, \infty)\end{array}$ & $\begin{array}{l}1 \\
1\end{array}$ & $\begin{array}{l}\{2,3\} \\
\{2,3\}\end{array}$ \\
\hline
\end{tabular}

Table 3. Semigroups of $(\mathbb{N},+)$ with the form $M(p)$.

5.4. For a given weight sequence $p=\left(p_{1}, \ldots, p_{t}\right)$, the Coxeter polynomials $f_{\left(p_{1}, \ldots, p_{t}\right)}(T)$ and $\hat{f}_{\left(p_{1}, \ldots, p_{t}\right)}(T)$ are readily computed by (4.1). In case Root $\hat{f}_{\left(p_{1}, \ldots, p_{t}\right)}(T) \subset \mathbb{S}^{1}$, then using (3.2), the Poincaré series $P_{C}(T)$ can be written as a rational function

$$
P_{C}(T)=\frac{\prod_{i=1}^{m}\left(1-T^{c_{i}}\right)}{\prod_{j=1}^{n}\left(1-T^{d_{j}}\right)}(1-T)^{r}
$$


for sequences $\left(d_{1}, \ldots, d_{n}\right)$ and $\left(c_{1}, \ldots, c_{m}\right)$ of natural numbers $\geq 2$ and some $r \in \mathbb{Z}$. In case $p$ is of wild type, then by (4.1)

$$
\hat{f}_{\left(p_{1}, \ldots, p_{t}\right)}(1)=-f_{\left[p_{1}, \ldots, p_{t}\right]}(1)=\left[(t-2)-\sum_{i=1}^{t} \frac{1}{p_{i}}\right] \prod_{i=1}^{t} p_{i}>0
$$

and by (5.1), $P_{C}(T)$ has a pole of order 2 at $T=1$, that is $m-n+r=-2$. Moreover, developing the series $P_{C}(T)$ we readily see that $r \neq 0$ implies that the semigroups $M(p)$ is $\mathbb{N}$, but [23, Th. 10.4] claims that $1+\left[\frac{1}{t-2}\right] \leq \alpha(p)$, that is, $\alpha(p)>1$ and therefore $r=0$. We state these considerations in the following.

Lemma. Let $p=\left(p_{1}, \ldots, p_{t}\right)$ be a weight sequence of wild type and $C=C(p, \lambda)$ be a canonical algebra. Then Root $\hat{f}_{\left(p_{1}, \ldots, p_{t}\right)}(T) \subset \mathbb{S}^{1}$ if and only if $R(p, \lambda)$ is formally n-generated, that is

$$
P_{C}(T)=\frac{\prod_{i=1}^{n-2}\left(1-T^{c_{i}}\right)}{\prod_{j=1}^{n}\left(1-T^{d_{j}}\right)}
$$

for numbers $c_{1}, \ldots, c_{n-2}$ and $d_{1}, \ldots, d_{n}$, all $\geq 2$, satisfying

$$
1+\sum_{j=1}^{n} d_{j}=\sum_{i=1}^{n-2} c_{i}
$$

Proof. If Root $\hat{f}_{\left(p_{1}, \ldots, p_{t}\right)}(T) \subset \mathbb{S}^{1}$, we showed that $P_{C}(T)$ has the desired form. Moreover,

$$
\sum_{j=1}^{n} d_{j}+\operatorname{deg} \hat{f}_{\left(p_{1}, \ldots, p_{t}\right)}(T)=\sum_{i=1}^{n-2} c_{i}+\operatorname{deg} f_{C}(T) .
$$

The converse follows from $\hat{f}_{\left(p_{1}, \ldots, p_{t}\right)}(T)=P_{C}(T) f_{C}(T)$.

In Table 2 , for a weight sequence $p=\left(p_{1}, \ldots, p_{t}\right)$ of wild type and $C=C(p, \lambda)$ the corresponding canonical algebra we have calculated (under the column 'Poincaré series') the sequences $\left(d_{1}, \ldots, d_{n}\right)$ and $\left(c_{1}, \ldots, c_{n-2}\right)$ corresponding to $P_{C}(T)=\frac{\hat{f}_{\left(p_{1}, \ldots, p_{t}\right)}(T)}{f_{\left(p_{1}, \ldots, p_{t}\right)}(T)}$.

5.5. Proof of Theorem [3. Let $C=C(p, \lambda)$ be a canonical algebra of wild type and $A=C[P]$ be an extended canonical algebra. Implications (a) $\Rightarrow(\mathrm{b}) \Rightarrow(\mathrm{c})$ are clear.

$(\mathrm{c}) \Rightarrow(\mathrm{a})$ : Assume Root $f_{A}(T) \subset \mathbb{S}^{1}$. By $(5.4), P_{C}(T)$ is $n$-generated. Hence $M(p)$ is at most $n$-generated. By Table 3 , if $n \geq 5$ then $p=\left(2,3, p_{3}\right)$ with $p_{3} \geq 11$. But a calculation shows (see Table 2 ) that Root $\hat{f}_{(2,3,11)}(T)$ is not contained in $\mathbb{S}^{1}$. Then 
Theorem 2 implies that Root $\hat{f}_{\left(2,3, p_{3}\right)}(T)$ is not contained in $\mathbb{S}^{1}$ for $p_{3} \geq 11$, which shows that $n \leq 4$.

For $k=\mathbb{C}$, the list entries in Table 2 correspond to Fuchsian singularities which are minimal elliptic as classified in [36]. These rings are graded complete intersection domains.

5.6. As a consequence of Theorem 3 and the classification given in Table 2 we get the following.

Corollary. Let $C=C(p, \lambda)$ be a canonical algebra of wild type. Let $A=C[P]$ be an extended canonical algebra. The following are equivalent:

(a) $\varphi_{A}$ is periodic.

(b) $\operatorname{Spec} \varphi_{A} \subset \mathbb{S}^{1}$ and $p$ is not $(3,3,3,3)$ or $(2,2,2,2,4)$.

(c) $f_{A}(T)=\prod_{i=1}^{m} \phi_{s_{i}}(T)^{e_{i}}$ for $1 \leq s_{1}<s_{2}<\cdots<s_{m}$ and $e_{i} \geq 1(1 \leq i \leq m)$, with $e_{m}=1$.

\section{Graded integral domains with 3 homogeneous generators}

6.1. The following simple remark is well-known.

Lemma. Let $R$ be a graded complete intersection integral k-algebra of Krull dimension 2. Then $R$ is generated by 3 homogeneous elements if and only if $R=$ $k\left[x_{1}, x_{2}, x_{3}\right] /(f)$ with $\operatorname{deg} x_{i}=d_{i}(1 \leq i \leq 3)$ and $f$ a homogeneous prime polynomial. In this case the Poincaré-Hilbert series of $R$ has the form

$$
\sum_{n=0}^{\infty}\left(\operatorname{dim}_{k} R_{n}\right) T^{n}=\frac{1-T^{c}}{\left(1-T^{d_{1}}\right)\left(1-T^{d_{2}}\right)\left(1-T^{d_{3}}\right)}
$$

for some natural numbers $c, d_{1}, d_{2}, d_{3}$ satisfying $1+d_{1}+d_{2}+d_{3}=c$.

Proof. Assume we have a graded surjection $g: k\left[x_{1}, x_{2}, x_{3}\right] \rightarrow R$ such that $y_{i}=g\left(x_{i}\right)$ is homogeneous of degree $d_{i}, 1 \leq i \leq 3$. Since $R$ is graded integral, then $I=\operatorname{ker} g$ is a prime ideal. Since $R$ has Krull-dimension two, the ideal $I$ has height one, hence it is principal. Let $I=(f)$ and $\operatorname{deg}(f)=c$. Then the Poincaré series has the desired form and $1+d_{1}+d_{2}+d_{3}=c$ by (4.4). 
6.2. Proof of Theorem 4. Let $C=C(p, \lambda)$ be a wild canonical algebra and $A=$ $C[P]$ a corresponding extended canonical algebra.

(a) $\Rightarrow$ (b): Assume $R(p, \lambda)$ is formally 3 -generated. By (5.4), Root $f_{A} \subset \mathbb{S}^{1}$. The result follows from the list given in Table 2 .

(b) $\Rightarrow$ (a): follows as above from Table 2 and (5.4).

Assume $R=R(p, \lambda)$ is formally 3 -generated with

$$
\sum_{n=0}^{\infty}\left(\operatorname{dim}_{k} R_{n}\right) T^{n}=\frac{1-T^{c}}{\left(1-T^{d_{1}}\right)\left(1-T^{d_{2}}\right)\left(1-T^{d_{3}}\right)}
$$

with $\left(d_{1}, d_{2}, d_{3}\right),(c)$ according to Table 2 . We shall consider two distinguished situations:

if $t=3$, then $R$ is a quasi-homogeneous complete intersection of the form $k\left[x_{1}, x_{2}, x_{3}\right] /(f)$ with $\operatorname{deg} x_{i}=d_{i}$ and $f$ a homogeneous relation as displayed in (6.3). The case $t \geq 4$ and $k=\mathbb{C}$ is considered in (6.4).

6.3. Theorem. [21]. Let $C=C(p, \lambda)$ be a canonical algebra of wild weight type $p=\left(p_{1}, p_{2}, p_{3}\right)$ such that the graded algebra $R=R(p, \lambda)$ is formally 3-generated. Then $R$ has the form

$$
R=k[x, y, z]=k[X, Y, Z] /(F)
$$

where the relation $F$, the degree triple $\operatorname{deg}(x, y, z)$ and $\operatorname{deg}(F)$ are displayed in $T a$ ble 4:

\begin{tabular}{c|c|c|c|c|c|} 
& $p$ & $\operatorname{deg}(x, y, z)$ & relation $F$ & $\operatorname{deg}(F)$ & Name \\
\hline Index $=6$ & $(2,3,7)$ & $(6,14,21)$ & $Z^{2}+Y^{3}+X^{7}$ & 42 & $E_{12}$ \\
& $(2,3,8)$ & $(6,8,15)$ & $Z^{2}+X^{5}+X Y^{3}$ & 30 & $Z_{11}$ \\
& $(2,3,9)$ & $(6,8,9)$ & $Z^{2}+X Z^{2}+X^{4}$ & 36 & $Q_{10}$ \\
\hline \hline \multirow{5}{*}{4} & $(2,4,5)$ & $(4,10,15)$ & $Z^{2}+Y^{3}+X^{5} Y$ & 30 & $E_{13}$ \\
& $(2,4,6)$ & $(4,6,11)$ & $Z^{2}+X^{4} Y+Z Y^{3}$ & 22 & $Z_{12}$ \\
& $(2,4,7)$ & $(4,6,7)$ & $Y^{3}+X^{3} Y+X Z^{2}$ & 18 & $Q_{11}$ \\
& $(2,5,5)$ & $(4,5,10)$ & $Z^{2}+Y^{2} Z+X^{5}$ & 20 & $W_{12}$ \\
& $(2,5,6)$ & $(4,5,6)$ & $X Z^{2}+Y^{2} Z+X^{4}$ & 16 & $S_{11}$ \\
\hline \hline 3 & $(3,3,4)$ & $(3,8,12)$ & $Z^{2}+Y^{3}+X^{4} Z$ & 24 & $E_{14}$ \\
& $(3,3,5)$ & $(3,5,9)$ & $Z^{2}+X Y^{3}+X^{3} Z$ & 18 & $Z_{13}$ \\
& $(3,3,6)$ & $(3,5,6)$ & $Y^{3}+X^{3} Z+X Z^{2}$ & 15 & $Q_{12}$ \\
& $(3,4,4)$ & $(3,4,8)$ & $Z^{2}-Y^{2} Z+X^{4} Y$ & 16 & $W_{13}$ \\
& $(3,4,5)$ & $(3,4,5)$ & $X^{3} Y+X Z^{2}+Y^{2} Z$ & 13 & $S_{12}$ \\
& $(4,4,4)$ & $(3,4,4)$ & $X^{4}-Y Z^{2}+Y^{2} Z$ & 12 & $U_{12}$ \\
\hline
\end{tabular}

Table 4. 
As observed in [21], these 14 equations are equivalent to Arnold's exceptional unimodal singularities. The equations are slightly different to those of the singularity theory classification, but equivalent for $k=\mathbb{C}$.

6.4. In view of the identification of $R(p, \lambda)$ with a ring of automorphic forms (5.5) in case $k=\mathbb{C}$, we get:

Theorem [9]. Let $C=C(p, \lambda)$ be a canonical algebra of wild type $p=\left(p_{1}, \ldots, p_{t}\right)$ with $t \geq 4$ over the complex numbers $\mathbb{C}$ and $R=R(p, \lambda)$ be the associated graded algebra. Then the following are equivalent:

(a) $R(p, \lambda)$ is formally 3-generated;

(b) $9 \leq \sum_{i=1}^{t} p_{i} \leq 11$

(c) there is a parameter sequence $\lambda^{\prime}=\left(\lambda_{3}^{\prime}, \ldots, \lambda_{t}^{\prime}\right)$ such that the algebra $R\left(p, \lambda^{\prime}\right)$ is of the form

$$
k[x, y, z]=k[X, Y, Z] /(F)
$$

where the relation $F$, the degree sequence $\operatorname{deg}(x, y, z)$ and $\operatorname{deg}(F)$ are displayed below:

\begin{tabular}{c|c|c|c|c|c|} 
& $p$ & $\operatorname{deg}(x, y, z)$ & relation $F$ & $\operatorname{deg}(F)$ & Name \\
\hline \multirow{4}{*}{$t=4$} & $(2,2,2,3)$ & $(2,6,9)$ & $Z^{2}+Y^{3}+X^{9}$ & 18 & $J_{3,0}$ \\
& $(2,2,2,4)$ & $(2,4,7)$ & $Z^{2}+X Y^{3}+X^{7}$ & 14 & $Z_{1,0}$ \\
& $(2,2,2,5)$ & $(2,4,5)$ & $Y^{3}+X Z^{2}+X^{6}$ & 12 & $Q_{2,0}$ \\
& $(2,2,3,3)$ & $(2,3,6)$ & $Z^{2}+Y^{4}+X^{6}$ & 12 & $W_{1,0}$ \\
& $(2,2,3,4)$ & $(2,3,4)$ & $Y^{2} Z+X Z^{2}+X^{5}$ & 10 & $S_{1,0}$ \\
& $(2,3,3,3)$ & $(2,3,3)$ & $Z^{3}+Y^{3}+X^{3} Y$ & 9 & $U_{1,0}$ \\
\hline \hline$t=5$ & $(2,2,2,2,2)$ & $(2,2,5)$ & $Z^{2}+Y^{5}+X^{5}$ & 10 & $N A_{0,0}^{1}$ \\
& $(2,2,2,2,3)$ & $(2,2,3)$ & $Y Z^{2}+Y^{4}+X^{4}$ & 8 & $V N A_{0,0}^{1}$ \\
\hline
\end{tabular}

Table 5 .

\section{References}

[1] V.I. Arnold, S. Gusejn-Zade and A. Varchenko. Singularities of differentiable maps. Monographs in Math 82 Birkhäuser (1985).

[2] M. Barot and H. Lenzing. One-point extensions and derived equivalences. J. Algebra 264 (2003) 1-5.

[3] A.I. Bondal. Representations of associative algebras and coherent sheaves. Math. USSR-Izv. 34 (1990), 23-42.

[4] A.I. Bondal and M.M. Kapranov. Enhanced triangulated categories. Math. USSR-Sb. 70 (1991), 93-107. 
[5] R. O. Buchweitz. Maximal Cohen-Macaulay modules and Tate-cohomology over Gorenstein rings. Unpublished manuscript (1987) $155 \mathrm{pp}$.

[6] I. V. Dolgachev. Automorphic forms and quasihomogeneous singularities. Functional Anal. Appl. 9 (1975) 149-151.

[7] W. Ebeling and C. T. C. Wall. Kodaira singularities and an extension of Arnold's strange duality. Compositio Mathematica 56 (1985) 3-77.

[8] W. Ebeling. The monodromy groups of isolated singularities of complete intersections. Lect. Notes Math. 1293, Springer, Berlin (1987).

[9] W. Ebeling. The Poincaré series of some special quasihomogeneous surface singularities. Publ. RIMS, Kyoto Univ. 39 (2003), 393-413.

[10] P. Gabriel. Des catégories abéliennes. Bull. Soc. Math. France 90 (1962), 323-448.

[11] W. Geigle and H. Lenzing. A class of weighted projective curves arising in representation theory of finite dimensional algebras. In: Singularities, representation of algebras and vector bundles. Lect. Notes Math. 1273, Springer, Berlin (1987) 265-297.

[12] W. Geigle and H. Lenzing. Perpendicular categories with applications to representations and sheaves. J. Alg. 144 (1991) 273-343.

[13] S.I. Gelfand and Yu. I. Manin. Methods of Homological Algebra. Springer, Berlin (2003).

$[14]$ D. Happel. Triangulated categories in the Representation Theory of finite dimensional algebras. London Math. Soc. Lect. Note Ser. 119 (1988).

[15] D. Happel, I. Reiten, S. Smalø, Tilting in abelian categories and quasitilted algebras, Mem. Amer. Math. Soc. 575 (1996).

[16] D. Happel. A characterization of hereditary categories with tilting object. Invent. Math. 144 (2001) 161-179.

[17] H. Kajiura, K. Saito and A. Takahashi. Matrix factorizations and representations of quivers II: Type ADE case. arXiv:math.AG:0511155v2 3 Apr 2006.

[18] B. Keller. On differential graded categories. Preprint 2006.

[19] H. Krause. The stable derived category of a noetherian scheme. Compositio Math. 141 (2005), $1128-1162$.

[20] H. Laufer. On minimally elliptic singularities. Ann. J. of Math. 99 (1977) 1257-1295.

[21] H. Lenzing. Wild canonical algebras and rings of automorphic forms. In: Finite dimensional algebras and related topics. Kluwer (1994) 191-212.

[22] H. Lenzing and H. Meltzer. Tilting sheaves and concealed-canonical algebras. In: Proceedings of ICRA VII. CMS Conf. Proc. 18 (1996) 455-473.

[23] H. Lenzing and J. A. de la Peña. Wild canonical algebras. Math. Z 224 (1997) 403-425.

[24] H. Lenzing and J. A. de la Peña. Coxeter polynomials and a Chebysheff recursion formula. To appear.

[25] H. Lenzing. Coxeter transformations associated with finite dimensional algebras. In: Progress in Math 173 (1999) 287-308.

[26] J. Milnor. On the 3 dimensional Brieskorn manifolds $m(p, q, r)$. In: Knots, groups and 3-manifolds; Pap. dedic. Mem. R.H. Fox (1975) 175-225.

[27] A. Neeman. Triangulated Categories. Annals of Mathematics Studies, 148. Princeton University Press, Princeton (2001).

[28] W. Neumann. Brieskorn complete intersections and automorphic forms. Invent. Math. 42 (1977) 285-293.

[29] D. Orlov. Triangulated categories of singularities and D-branes in Landau-Ginzburg models. Proc. Steklov Inst. Math. 246 (2004) 227-248.

[30] D. Orlov. Derived categories of coherent sheaves and triangulated categories of singularities. arXiv:math.AG/0503632v2 22 Sep 2005. 
[31] J.A. de la Peña. On the dimension of the module-varieties of tame and wild algebras. Comm. in Algebra 19 (6), (1991) 1795-1807.

[32] J. Rickard. Morita theory for derived categories. J. London Math. Soc. 39 (1989), 436-456.

[33] C. M. Ringel. The canonical algebras. In: Topics in Algebra, part I. Banach Center Pub. 26 (1950) 407-432.

[34] C. M. Ringel. Tame algebras and integral quadratic forms. Lect. Notes Math., Springer, Berlin 1099 (1984).

[35] K. Ueda. Homological mirror symmetry and simple elliptic singularities. arXiv:math.AG/0604361 v2 3 Jul 2006.

[36] P. Wagreich. Automorphic forms and singularities with $\mathbb{C}^{*}$-action. Illinois J. Math. 25 (1981) 359-382.

[37] P. Wagreich. Algebras of automorphic forms with few generators. Trans. Amer. Math. Soc. 262 (1980) 367-389.

H. Lenzing

Institut für Mathematik

Universität Paderborn

33095 Paderborn

Germany

helmut@math.uni-paderborn.de
J.A. de la Peña

Instituto de Matemáticas

Universidad Nacional Autónoma de México

Ciudad Universitaria

México 04510, D.F.

México

jap@matem.unam.mx 